\title{
A recent origin for Saturn's rings from the collisional disruption of an icy moon
}

\author{
John Dubinski ${ }^{1}$ \\ Canadian Institute for Theoretical Astrophysics, 60 St. George St., University of Toronto, Toronto, Ontario, Canada M5S 3H8
}

\begin{abstract}
The disruption of an icy moon in a collision with an interloping comet a few hundred million years ago is a simple way to create Saturn's rings. A ring parent moon with a mass comparable to Mimas could be trapped in mean motion resonance with Enceladus and Dione in an orbit near the current outer edge of the rings just beyond the Roche zone. I present collisional N-body simulations of cometary impacts that lead to the partial disruption of a differentiated moon with a rocky core and icy mantle. The core can survive largely intact while the debris from the mantle settles into a ring of predominantly ice particles straddling the orbital radius of the parent moon. The nascent ring spreads radially due to collisional viscosity while mass re-accretes onto the remnant rocky core to form a new moon that can be identified as Mimas. The icy debris that migrates into the Roche zone evolves into Saturn's ring system. Torques from tidal interaction with Saturn and resonant interactions with the rings push the recently formed Mimas outward to its current position on the same timescale of a few hundred million years. This scenario accounts for the high ice fraction observed in Saturn's rings and explains why the ring mass is comparable to the mass of Mimas. The prior existence of a ring parent moon in mean motion resonance results in a tidal heating rate for Enceladus in the recent past that is significantly larger than the current rate.
\end{abstract}

Keywords: Saturn, rings, satellites; Satellite, dynamics; Enceladus; Mimas

\section{Introduction}

The origin of Saturn's rings has been an ongoing puzzle since Galileo's discovery of the system four hundred years ago. Current theories that explain the rings' origin are constrained by the mass, composition and age of the rings (Harris, 1984; Dones, 1991; Charnoz et al., 2009a, Canup, 2010, Charnoz et al., 2009b, Cuzzi et al., 2010) (See Charnoz et al. (2018) for a comprehensive review of the origin of planetary rings.) Observations of density waves in the $\mathrm{A}$ and $\mathrm{B}$ rings and comparisons of simulations of optical depth to Cassini observations for the denser B ring find a total mass of the rings comparable to Saturn's moon Mimas (Esposito et al., 1983; Robbins et al., 2010, Hedman and Nicholson, 2016) with uncertainties due to clumpiness of matter in the rings leading to a range varying from $2-10 \times 10^{19} \mathrm{~kg}$ cf. $M_{\text {Mimas }}=3.75 \times\left. 10^{19} \mathrm{~kg}\right|^{2}$ The rings are mainly composed of ice with historical radio and radar observations suggesting an ice mass fraction greater than 90\% (Cuzzi et al., 2010). The latest microwave observations from Cassini and the VLA imply ice mass fractions greater than 99\% for the A and B rings and slightly less for the $\mathrm{C}$ ring (Zhang et al. 2017a b, 2019) These measurements are inconsistent with the mass-weighted average value of $60 \%$ ice/40\% rock for Saturn's 5 classical inner moons (Matson et al. 2009). If the rings and moons form coevally in Saturn's early history and are born from a massive spreading ring (Charnoz et al., 2009b; Crida and Charnoz, 2012), one needs to explain their different compositions. One proposed solution to this problem is a scenario in which a differentiated moon with a mass comparable to Titan falls into Saturn at early times and loses its icy mantle via tidal stripping to form a nearly pure ice ring (Canup 2010). The final constraint on ring origin theories comes from the

\footnotetext{
1e-mail: dubinski@cita.utoronto.ca

${ }^{2}$ At the Cassini Science Symposium in August 2018, Luciano Iess presented results giving estimates for the total mass of the A, B, and C rings based on Cassini close flybys as $0.41 \pm 0.04 M_{\text {Mimas }}$ or $1.5 \times 10^{19} \mathrm{~kg}$ putting it at the low end of the range of previous estimates; http: //lasp.colorado.edu/media/projects/cassini/meetings/css2018/4-THURSDAY/3-Saturn_1_(S1-)/6-Iess-REVISED.pdf
} 
age of the rings. Two arguments suggest that the rings may be as young as a few hundred million years causing problems for theories requiring a primordial origin. The viscosity arising from dissipative particle collisions implies that a ring will spread to conserve angular momentum while losing orbital energy (Goldreich and Tremaine, 1982). The viscous timescale is $t_{v} \sim \Delta R^{2} / v$ where $\Delta R$ is the current radial width of the rings and $v$ is the viscosity with the underlying assumption that the ring originates as a radially thin object. This timescale is a few hundred million years based on the current estimates of ring viscosity for the A ring at least (Esposito, 1986, Daisaka et al., 2001). The more massive B ring could be much older based on similar arguments and more detailed treatments of the sources of viscosity in the denser B ring may imply longer evolution and survival times with the rings perhaps as old as the age of the Solar System supporting primordial origin theories (Daisaka et al., 2001, Salmon et al., 2010). Meteoroid bombardment can also change the composition of the rings over time through contamination from mixing with non-icy constituents. Modeling of the spectral evolution of the rings under exposure to meteoroids also implies a young age of only a few hundred million years again (Durisen et al., 1992; Cuzzi and Estrada, 1998; Estrada et al. 2015) while new results from Cassini (e.g., Zhang et al., 2017b) imply exposure ages in the range of 30-150 Myr. These results are confounding to primordial ring origin theories. Scenarios where the rings might form recently include collisional disruption of a moon or moons within the Roche zone through impacts with infalling comets (Harris, 1984) and tidal stripping of a massive icy Kuiper belt object passing close to Saturn (Dones, 1991; Hyodo et al., 2017). Both scenarios can happen at any time but would most likely occur during the Late Heavy Bombardment (Charnoz et al., 2009b). The collisional disruption theory is problematic since it is hard to form or move moons near the Roche zone as well as hold them in place due to tidal evolution. The tidal stripping theory seems unlikely to have occurred in recent times because of the requirement of a large Kuiper belt object - perhaps as massive as Pluto - having a near collision with Saturn. A complete collisional disruption requires a parent object that is nearly pure ice - a fact inconsistent with the composition of the icy moons. However, in a tidal disruption model the icy mantle of a differentiated parent object might be stripped to create an icy ring (Hyodo et al., 2017). The recent determination of small tidal dissipation factor $Q$ for Saturn (Lainey et al., 2012, 2017) implies another possible scenario where orbital instabilities can be driven by the more rapid tidal evolution of the icy satellite system. This could lead to moon-moon collisions within the past $10^{8}$ years that formed the current icy moon system and rings (Ćuk et al. 2016). Hyodo and Charnoz (2017) have explored this scenario and show that the debris from a hypothetical collision between a proto-Rhea and proto-Dione at $a=500000 \mathrm{~km}$ quickly reaccretes into other moons before spreading into the Roche zone making this scenario unlikely as a way to form the rings though more work needs to be done to understand this process. In summary, the inferred mass and composition of the rings create tension between theories that propose a primordial and recent origin and there is no strong consensus on a formation mechanism.

In this paper, we propose a modified version of the collisional disruption mechanism of ring formation (Harris, 1984) that is consistent with the mass and composition of the rings. In this model, a comet on a heliocentric orbit an Oort-cloud comet or Centaur - with sufficient mass and impact energy hits and disrupts a ring parent moon. The resulting debris then spreads along the moon's orbit to form the ring system. This mechanism is likely relevant to the formation of the less massive rings of Jupiter, Uranus and Neptune but faces difficulties in making Saturn's rings (Colwell, 1994) because of their relatively large mass and high ice fraction. The composition of a hypothetical ring parent moon for Saturn should be consistent with those of Saturn's moons shown in Table 11 (Matson et al., 2009. Thomas 2010). Since the mass of the rings is comparable to the mass of Saturn's moon Mimas (Robbins et al., 2010), the disrupted moon must be at least this large and one further needs to find a way to create a ring made of nearly pure ice. The ice fractions of Saturn's 5 mid-sized moons vary from 43\% (Enceladus) to 94\% (Tethys) with an mass-weighted average value of about $60 \%$ (Thomas, 2010) (see Table 1). The complete disruption of a moon with the current average composition of the extant moons would create a ring inconsistent with the observations though perhaps a parent moon with the composition of Tethys might be adequate.

A further problem with this collisional scenario arises when considering the initial orbital radius of a hypothetical ring parent moon. To remain stable in the presence of Saturn's tidal forces, the parent moon must be located outside of the Roche $(1847)$ radius $R_{\text {Roche }}=2.45 R_{S}\left(\rho_{S} / \rho_{\text {moon }}\right)^{1 / 3}$ where $R_{S}$ and $\rho_{S}$ are the radius and mean density of Saturn and $\rho_{\text {moon }}$ is the moon's density. However, the parent moon cannot be too far removed from the Roche radius so that some of the collisional debris can migrate inwards to form the rings before reassembling into a new moon. For a moon composed of pure ice, $R_{\text {Roche }} \approx 130000 \mathrm{~km}$ consistent with the rings current radial extent of $R<137000 \mathrm{~km}$, so the parent moon should ideally located near this radius. The co-orbital moons Janus and Epimetheus are already near the current edge of the rings but their collisional disruption would create a ring with a mass that is an order of magnitude 


\begin{tabular}{rrrrr}
\hline Object & $\mathrm{a}\left(10^{5} \mathrm{~km}\right)$ & Mass $\left(10^{20} \mathrm{~kg}\right)$ & $\bar{\rho}\left(\mathrm{g} / \mathrm{cm}^{3}\right)$ & Ice Fraction \\
\hline A Ring & $1.22-1.37$ & 0.06 & - & $90-95 \%$ \\
B Ring & $0.92-1.18$ & $0.2-1.0$ & - & $90-95 \%$ \\
Janus & 1.51 & 0.020 & 0.63 & - \\
Mimas & 1.85 & 0.375 & 1.15 & $74 \%$ \\
Enceladus & 2.38 & 1.08 & 1.61 & $43 \%$ \\
Tethys & 2.95 & 6.17 & 0.99 & $94 \%$ \\
Dione & 3.77 & 10.95 & 1.48 & $50 \%$ \\
Rhea & 5.27 & 23.07 & 1.24 & $67 \%$ \\
\hline Ring parent & 1.40 & 0.750 & 1.03 & $84 \%$ \\
\hline
\end{tabular}

Table 1: The properties of Saturn's rings (Robbins et al. 2010, Cuzzi et al. 2010 Hedman and Nicholson 2016) and mid-sized moons (Matson et al. 2009 Thomas 2010, compared to a hypothetical ring parent moon. Theories based on a coeval origin of the rings and moons have difficulty in explaining a high ice mass fraction in the rings compared to the moons. The properties of the proposed ring parent moon are included for comparison.

too small. Assuming the disrupting parent moon is massive enough, the debris would settle into a ring promptly and then spread radially on the viscous timescale discussed above. If the disruption occurred recently - say within the past $500 \mathrm{Myr}$, the primordial existence of a Mimas-sized moon located just near the Roche radius might seem unlikely since tidal friction (e.g., Goldreich and Soter, 1966) would have caused a moon of this mass to migrate away from this position to Mimas's current orbital radius within the age of the solar system based on the conventional values of the Saturn's Love number $k_{2, S}$ and tidal dissipation function $Q_{S}$ having a ratio $k_{2, S} / Q_{S}=2.0 \times 10^{-5}$. Recent analysis of a century of astrodynamical measurements combined with Cassini's observations of Saturn's moons imply values $k_{2, S} / Q_{S} \approx 1.6 \times 10^{-4}$ reducing the tidal evolution timescale by an order of magnitude making it even more difficult to consider a moon existing at this position for long (Lainey et al., 2012, 2017) and in fact implying short timescales for potential catastrophic orbital instabilities for the entire inner moon system (Ćuk et al., 2016). However, Fuller et al. (2016) have argued that Saturn's effective $Q$ may be intermittently diminished by resonant locking between dynamical tidal oscillations commensurate with orbital frequencies of satellites while the long term average of $Q_{S}$ would be closer to the conventional value. The implications of a evolving value of $Q_{S}$ have not been fully explored. The only way to hold a moon in place near the Roche radius over the age of the solar system prior to a recent disruption is via an $e$-type mean motion resonant (MMR) trapping with the other icy moons. Another difficulty is that such destructive events may be rare with the disruption of a ring parent moon of the mass of Mimas happening once every $20 \mathrm{Gyr}$ at the current cometary flux rate giving a probability of destruction in the last $500 \mathrm{Myr}$ of $\sim 2 \%$ (Lissauer et al. 1988 , Dones et al. 2009: Charnoz et al., 2009a). The rarity of an event in itself does not necessarily rule out its occurrence if no alternative more probable mechanisms can be found.

The scenario proposed in this paper overcomes the above difficulties and provides a modified collisional scenario for a recent origin of Saturn's rings. The strange fact that the mass of the rings is close to the mass of Mimas may not be a coincidence but rather a clue pointing to a collisional disruption origin ${ }^{3}$ Now consider the collisional disruption of a ring parent moon that is twice the mass of Mimas located just outside the current edge of the rings near the Roche radius at a radius $a \approx 140000 \mathrm{~km}$. After disruption, half of the collisional debris migrates inwards within the Roche zone to become a new ring while the remainder of the debris migrates outwards along with any remnant of the collision and likely reassembles to become a new moon following the mechanism described by Crida and Charnoz (2012). One might identify this new moon with Mimas itself. In this picture, Mimas forms coevally with the rings and migrates to its current position from the time the rings formed.

To support this hypothesis, imagine taking the total mass of Saturn's A and B rings and Mimas itself and placing it within a single body - a hypothetical ring parent moon composed of mass from the rings and Mimas. Using reasonable estimates of the ring surface density (e.g., Robbins et al. 2010) and assuming there is no significant mass loss from the Saturn system during the disruption, one can calculate the total mass and angular momentum of this combined

\footnotetext{
${ }^{3}$ It should be noted that primordial origin theories that posit more complex viscosity evolution for the rings also result in a ring with a mass comparable to Mimas (Salmon et al. [2010).
} 
system and compute the orbital radius $a_{\text {parent }}$ for this hypothetical ring parent moon:

$$
a_{\text {parent }}=\left(G M_{S}\right)^{-1}\left(\frac{J_{A}+J_{B}+J_{M I M A S}}{M_{A}+M_{B}+M_{M I M A S}}\right)^{2}
$$

Assuming a uniform density $\Sigma_{\text {ring }}$ with radial range $R_{0}<R<R_{1}$, the ring mass and angular momentum are:

$$
\begin{aligned}
M_{\text {ring }} & =\pi \Sigma\left(R_{1}^{2}-R_{0}^{2}\right) \\
J_{\text {ring }} & =\frac{4 \pi}{5}\left(G M_{S}\right)^{1 / 2} \Sigma\left(R_{1}^{5 / 2}-R_{0}^{5 / 2}\right)
\end{aligned}
$$

For Saturn's $A$ and $B$ rings, the analysis of Robbins et al. (2010) and Hedman and Nicholson (2016) sets limits on the ring surface densities with $\Sigma_{A}=420-520 \mathrm{~kg} / \mathrm{m}^{2}$ and $\Sigma_{B}=400-4800 \mathrm{~kg} / \mathrm{m}^{2}$ though earlier estimates by Esposito et al. (1983) give $\Sigma_{A}=500 \mathrm{~kg} / \mathrm{m}^{2}$ and $\Sigma_{B} \approx 1000 \mathrm{~kg} / \mathrm{m}^{2}$. With the A and B ring radial ranges of $122000-137000 \mathrm{~km}$ and 92000 - 118000 one can compute a range of ring masses and angular momenta using the equations above. Using Mimas's mass and orbital radius of $M_{M I M A S}=3.75 \times 10^{19} \mathrm{~kg}$ and $a_{M I M A S}=185400 \mathrm{~km}$ once can find the orbital angular momentum of Mimas and so determine a mass and orbital radius for a ring parent moon from equation 1 . The result is $M_{\text {parent }}=1.3-3.4 M_{\text {MIMAS }}$ with $a_{\text {parent }}=128000-167000 \mathrm{~km}$ with the lower mass estimates corresponding to the larger orbital radii. Note that the mass of Enceladus is $M_{E N C E L A D U S}=2.9 M_{M I M A S}$ so the proposed ring parent moon is consistent with the properties of the innermost moons. This orbital radius straddles the Roche radius defining the edge of the rings so in principle an ice+rock moon could be located in this position. This orbital radius is also fortuitously very close to the current 4:2:1 mean motion resonant orbital radius with Enceladus and Dione located at $a \approx 150000 \mathrm{~km}$. Mean motion resonances between satellites are common in the solar system and multiple resonances have a precedent in Jupiter's moons with Io, Europa and Ganymede (Goldreich, 1965) arranged in the same 4:2:1 hierarchy as originally observed by Laplace. A reasonable hypothesis is that a similar resonant arrangement occurred with a ring parent moon, Enceladus and Dione when the inner icy moons of Saturn formed. Such a system of icy moons may have formed by the viscous spreading of a primordial massive ring composed of ice and rock in the early phases of the formation of Saturn and its mid-sized moons (Canup, 2010, Charnoz et al., 2010). Perhaps the last of this primordial ring material ended up in a moon just beyond the Roche radius. If this hypothetical ring parent moon was trapped in resonance in the past, its outward tidal migration would be slowed down significantly and it could remain in this vulnerable position prior to a collision that would eventually recreate a ring in recent times.

A final requirement for this scenario to work is a mechanism that leads to the nearly pure icy composition of Saturn's rings. The hypothetical parent moon is likely differentiated from the strong tidal heating expected from close proximity to Saturn and resonant interactions with Enceladus and Dione. If we imagine a parent moon that is twice the mass of Mimas with the same rock mass then its ice fraction is $84 \%$, a plausible value ranking it between Mimas and Tethys. One might expect a partial disruption to liberate the mantle to form ice rings while leaving behind the rocky core that migrates outward within a newly forming Mimas or small moon that subsequently interacts with Mimas.

In this paper, we explore this collisional scenario using simulations of hypervelocity cometary impacts with a hypothetical differentiated ring parent moon located near Saturn's Roche radius. We first describe methods based on a new collisional N-body rubble pile code using simulations with $10^{7}$ particles. We present results that show how a ring of nearly pure ice can be created along with a remnant rocky moon straddling the Roche radius. We argue that the subsequent evolution of this system can plausibly transform into Saturn's ring system and a recently formed Mimas over a few hundred million years. We also discuss the implications of this scenario for the rest of Saturn's inner satellite system.

\section{Methods}

\subsection{Collisional N-body methods}

We model the moon as system of gravitating hard colliding spheres using a new collisional N-body code based upon a parallelized N-body treecode originally developed for galactic dynamics (Dubinski, 1996). The code has been modified to follow collisions between particles treated as hard spheres and has similarities to other codes used to model rubble piles (e.g., Richardson et al., 2000). The new code uses a parallelization strategy that permits the simulation of $\mathrm{N}$-body systems with as many as $10^{8}$ particles on modern parallel supercomputers. We describe the code in detail in Appendix A. 


\subsection{Ring parent moon}

We model the ring parent moon as a rubble pile of $N$ frictionless hard spheres of equal radius. Following similar methods applied to models of asteroids (e.g., Leinhardt et al., 2000), we set up systems of hard spheres within an initially spherical distribution with small random velocities. For sufficiently large numbers of particles, impacts with rubble pile simulations behave like a fluid described by a hard-sphere equation of state (EOS) and therefore can be used as a proxy for more computationally difficult hydrodynamics simulations using equations of state for ice and rock. We justify this approximation for our problem in more detail below. We describe the methods for setting up equilibrium gravitating systems of colliding hard spheres based on a hard-sphere EOS in Appendix B.

The ring parent moon model consists of $10 \mathrm{M}$ hard colliding spheres divided between a rocky core containing $500 \mathrm{~K}$ particles and a icy mantle containing $9.5 \mathrm{M}$ particles. The particles within the rocky core have masses three times those of the icy mantle so that mean densities of rock and ice are $\rho_{\text {rock }}=2.8 \mathrm{~g} / \mathrm{cm}^{3}$ and $\rho_{\text {ice }}=0.935 \mathrm{~g} / \mathrm{cm}^{3}$ - the density of ice at the ambient temperature of $T=70 \mathrm{~K}$ near Saturn. The total mass of the moon is $M_{\text {parent }}=2 M_{M I M A S}$ with the core mass intentionally set to be the same value as the implied mass of rock within Mimas based on its mean density of $\rho=1.15 \mathrm{~g} / \mathrm{cm}^{3}$. The radius of the ring parent moon is $R=259 \mathrm{~km}$ leading to a mean density of $\rho=1.03 \mathrm{~g} / \mathrm{cm}^{3}$ and ice fraction of $84 \%$. The density profile of our model is presented in Figure 1 . The particle radii for this model are $R=1.05 \mathrm{~km}$.

The moon is placed in a circular orbit at $a=140000 \mathrm{~km}$ with an orbital velocity $v_{m}=16.5 \mathrm{~km} / \mathrm{s}$ and period $P=14.9 \mathrm{hrs}$ about Saturn. For a moon this close to Saturn, one expects significant tidal flattening and the moon should take the form of a Roche ellipsoid. In principle, the exact shape of a Roche ellipsoid can be computed for homogeneous bodies. Since our model is differentiated, we find the equilibrium configuration by setting the initially spherical model described above in synchronous rotation on its orbit and letting it relax dynamically within Saturn's tidal field over one orbit. The final Roche ellipsoid has an surface axis ratio a:b:c=1:0.69:0.65 and major axis length of $a=320 \mathrm{~km}$ while the inner rocky core remains nearly spherical (Figure 2).

\subsection{Comet}

We similarly construct a model comet as a spherical rubble pile made of 5000 ice particles. The comet's mass is $M_{c}=3.75 \times 10^{16} \mathrm{~kg}$ with a radius $R=22 \mathrm{~km}$ comparable to the size and mass of Comet Hale-Bopp (Weaver and Lamy, 1997). Below we show that this mass is sufficient to disrupt a ring parent moon near the Roche zone. The comet might come from the Oort cloud or the Centaur population. For a zero-energy heliocentric orbit, the excess speed at Saturn is $v_{\infty}=14 \mathrm{~km} / \mathrm{s}$. Centaur comets originating from Kuiper belt objects (KBOS) on Neptune crossing orbits have a median excess speed of approximately $v_{\infty}=3 \mathrm{~km} / \mathrm{s}$ as determined from simulations (see Figure 1 of Levison et al. (2000)). The comet speed before impact is $v_{c}=\left(v_{e}^{2}+v_{\infty}^{2}\right)^{1 / 2} \approx 23-27 \mathrm{~km}$ where $v_{e}$ is the escape velocity at the orbital radius $a$. With the moon's orbital velocity of $v_{m}=16.5 \mathrm{~km} / \mathrm{s}$, the relative velocity of impact $v_{\text {rel }}$ is in the range $7<v_{r e l}<44 \mathrm{~km} / \mathrm{s}$. In this paper, we consider three collisional trajectories corresponding to the case of a rear-end, side-on and head-on collisions with relative velocities corresponding to comets on zero-energy orbits with $v_{r e l}=11,27$ and $44 \mathrm{~km} / \mathrm{s}$ to explore a range of impactor energies. In all three cases, the impacts are direct with no inclination to the surface and in the plane of the parent moon's orbit. In general, the comet could be coming in from any direction with a range of impact parameters and velocities. The goal of this paper is to demonstrate the plausibility of this mechanism for creating rings. A more thorough study using a range of impact geometries and energies will follow once the mass of the rings determined from the Cassini ring flybys is known.

\section{Collisional disruption}

An impacting comet deposits kinetic energy explosively at the surface of the target moon creating an inward propagating shockwave that leads to complete or partial disruption of the moon (e.g., Holsapple, 1993). Collisional disruption is quantified by the parameter $Q_{D}^{*}$, the energy per unit mass of the target required to unbind half of the total mass (Benz and Asphaug, 1999). For isolated pure ice targets, simulations show the scaling relation $Q_{D}^{*}=$ $0.05(R / \mathrm{m})^{1.188} \mathrm{~J} / \mathrm{kg}$ where $R$ is the radius of the target in meters (Movshovitz et al., 2015). The typical mass of an impacting comet $M_{c}$ with relative velocity $v_{r e l}$ that will disrupt the moon is found by equating the disruption energy to the kinetic energy

$$
M_{c}=\frac{2 M_{\text {moon }} Q_{D}^{*}}{v_{r e l}^{2}} \text {. }
$$




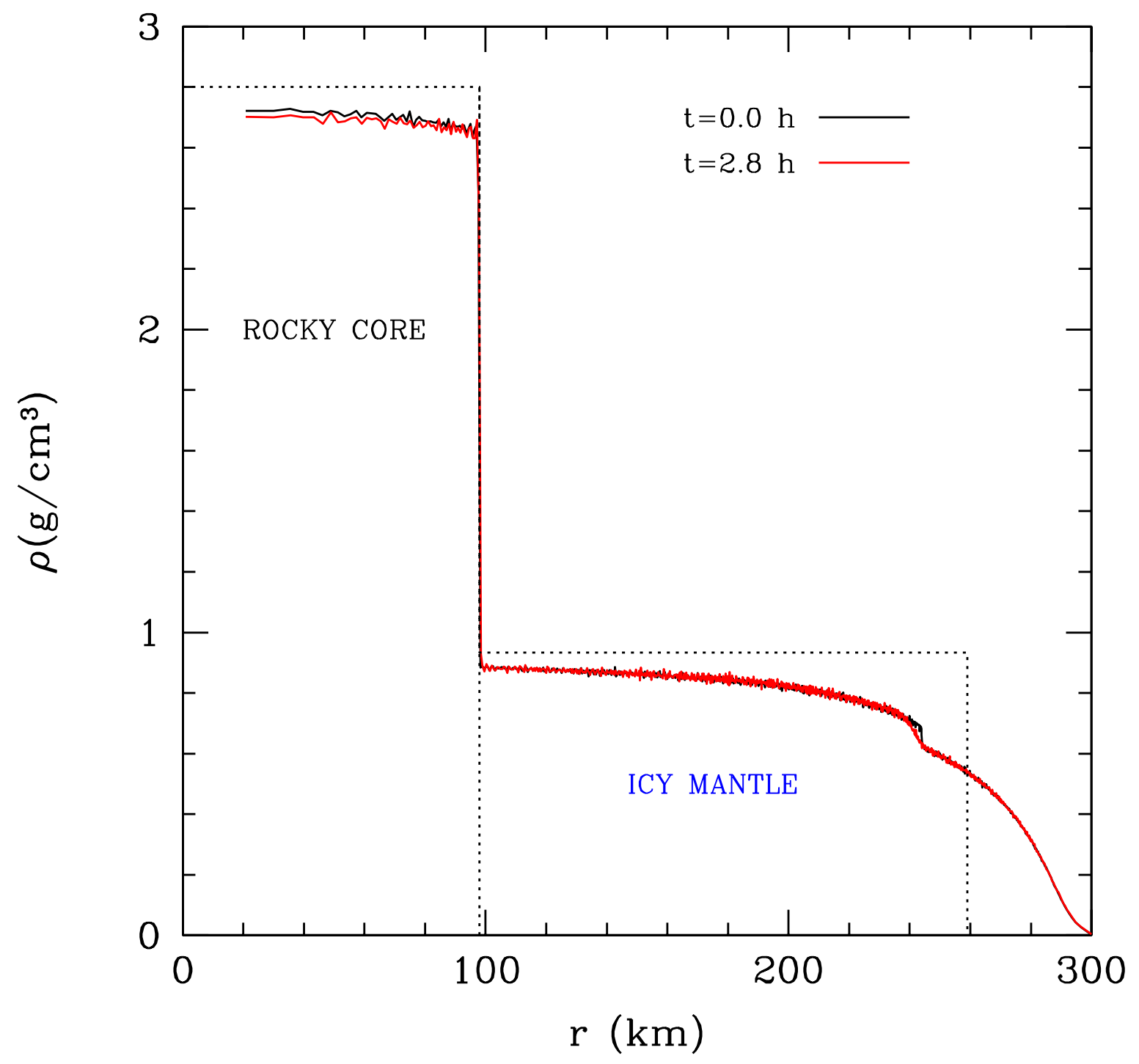

Figure 1: The density profile of the ring parent moon model. The moon has a rocky core with radius $R=100 \mathrm{~km}$ and an icy mantle extending to $R=300 \mathrm{~km}$. The dashed line describes the idealized density for a moon with a homogeneous rocky core and icy mantle. The solid black line describes the density profile for the hydrostatic equilibrium calculated from the hard-sphere equation of state with velocity dispersions of $\sigma=6 \mathrm{~m} / \mathrm{s}$ in the core and $\sigma=11 \mathrm{~m} / \mathrm{s}$ in the mantle. The red line describes the density profile after the model has been dynamically evolved for 2.8 hours showing that the model is almost in exact equilibrium. The discontinuity in density at $R=240 \mathrm{~km}$ is due to the phase change from a hexagonally close packed crystal solid to a more random glass-like fluid phase. 


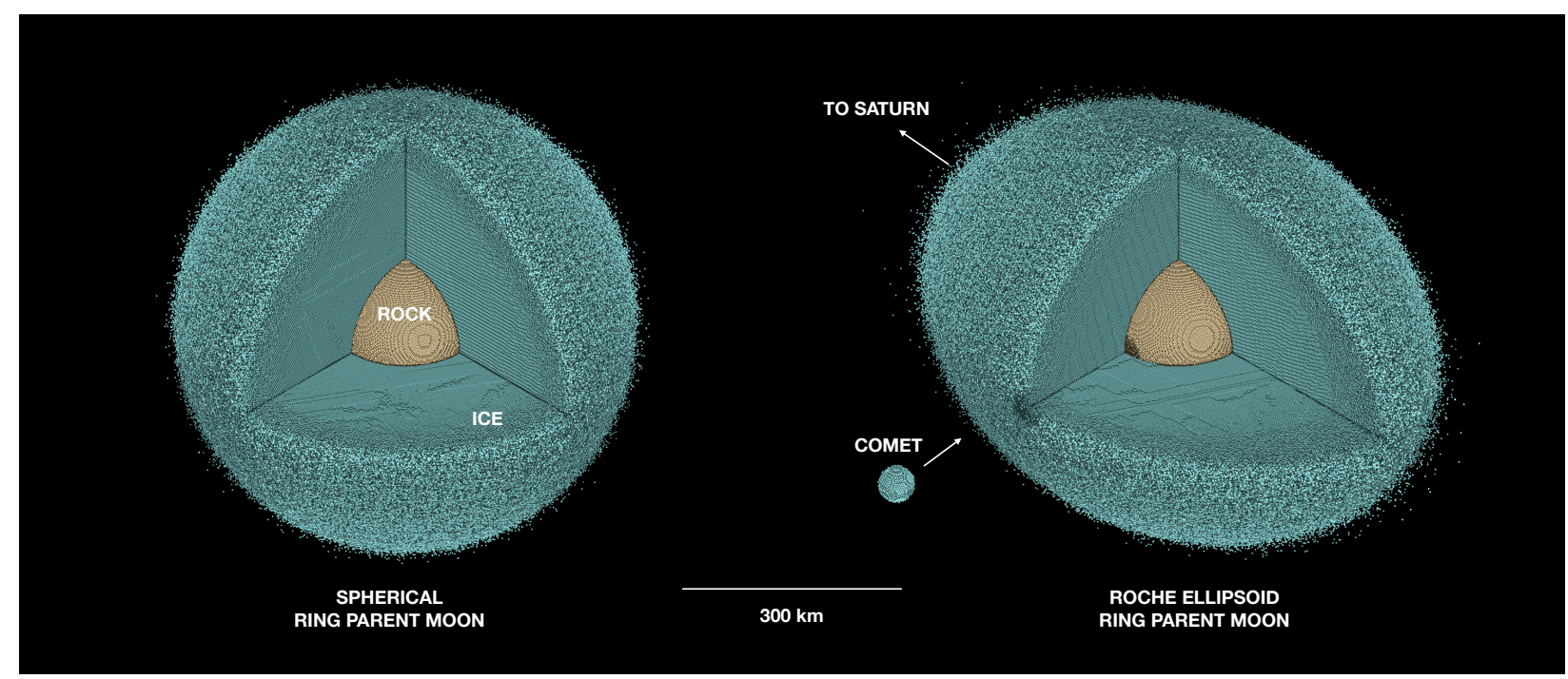

Figure 2: A cutaway rendering of the spherical differentiated ring parent moon and the tidally adjusted Roche ellipsoid model with the comet impactor to scale. The moon model is composed of a rocky core containing $520 \mathrm{~K}$ particles and icy mantle made of $9.5 \mathrm{M}$ particles. All particles have a radius $R=1.05 \mathrm{~km}$. The rock particles (orange) are $3 \times$ the mass of the ice particles (blue). The comet is made of 5000 ice particles. The moon has a total mass $M_{\text {moon }}=2 M_{\text {MIMAS }}$ while the comet has a mass $M_{c}=10^{-3} M_{M I M A S}$.

A typical relative velocity is $27 \mathrm{~km} / \mathrm{s}$ as discussed above requires a pure ice comet impactor mass, $M_{c} \approx 4 \times 10^{16} \mathrm{~kg}$ with radius $R \approx 22 \mathrm{~km}$ to disrupt a ring parent moon of twice the mass of Mimas. We use a comet mass comparable to this value in our simulations.

The use of a collisional N-body code to follow this process needs to be justified since hypervelocity disruptive impacts are complex and are usually treated with a hydrodynamics code using equations of state for ice and rock that span behavior from the solid state through melting to vaporization (e.g., Benz and Asphaug, 1999, Kraus et al., 2011). Collisional particle simulations do not treat the detailed hydrodynamics and shock-heating induced phase transitions at the impact point but we argue here that their application is a reasonable approximation since the bulk of the energy goes into simply unbinding and shattering the moon. One expects the shockwave from the impact to lead to the melting and vaporization of some of the target mass for impact velocities $>10 \mathrm{~km} / \mathrm{s}$ (Kraus et al., 2011). For ice-on-ice hypervelocity collisions, the expected mass of melt plus vapor is $\sim 100$ times the mass of the impactor (see Fig. 6 in Kraus et al. (2011)). The relatively small mass of the impactor in these simulations implies that around 5\% of the mass of the moon is melted or vaporized. While collisional N-body simulations miss the details of the complex behavior at the point of impact, most of the impact energy goes into disrupting the moon rather than melting and vaporizing it. As the pressure wave from impact propagates away from the impact point, the overpressure exceeds the local hydrostatic pressure and causes the moon to unbind. A solid icy moon with a rocky core therefore shatters into fragments and depending on the impact energy, the moon can completely or partially unbind. For lower impact energies, a fraction of the mass can remain bound after the impact. The process of shattering is also not well-defined and depends on the structural properties of the moon (Ballouz et al. 2015) but in this rubble-pile approximation the moon simply breaks up into millions of equal mass fragments represented by the particles.

These models use $10^{2-3}$ times as many particles as typically used in rubble pile collisions and SPH simulations and so permit a small impactor to target mass ratio. In hypervelocity collisions with rubble piles, it is necessary to use small enough timesteps to accurately distribute the impact energy throughout the target body and so conserve energy through the disruption process. Typically, the initial timestep needs to be $\delta t \approx R_{p} / v$ where $R_{p}$ is the radius of the particles and $v$ is the relative velocity at impact. For our largest simulations, $R_{p}=1.05 \mathrm{~km}$ and $v<44 \mathrm{~km} / \mathrm{s}$ the initial $\delta t \sim 0.02 s$. Once the impact energy has distributed throughout the target body, the particle collision rate drops off as the density decreases and the timestep can be increased to speed up the calculation.

To validate this approach, we carried out a study of 20 isolated disruptive collisions of the putative ring parent moon using models composed of $1 \mathrm{M}$ particles with a range of impact energies varying both the mass ratio and relative 


\begin{tabular}{rrrrr}
\hline Collision & $v_{\text {rel }}(\mathrm{km} / \mathrm{s})$ & $Q_{D}\left(10^{5} \mathrm{~J} / \mathrm{kg}\right)$ & $M_{\text {remnan }, i} / M_{\text {Mimas }}$ & Ring ice fraction \\
\hline rear-end & 11.2 & 0.3 & 1.02 & 1.0 \\
side-on & 22.4 & 1.2 & 0.74 & 1.0 \\
head-on & 44.2 & 4.9 & 0.16 & 0.91 \\
\hline
\end{tabular}

Table 2: The mass of the comet is $m_{\text {comet }}=3.75 \times 10^{16} \mathrm{~kg}=5 \times 10^{-4} m_{\text {moon }}$ and it is $100 \%$ ice. The remnant mass is measured at the end of the simulation. The rocky core remains intact for the rear-end and side-on collisions while about half of the rocky core in the head-on collision ends up in the ring. One expects the remnant to accrete some of the mass of the nascent ring as it spreads. The optimal impact energy for creating Mimas with the right mass is somewhere between $1.2-4.9 \times 10^{5} \mathrm{~J} / \mathrm{kg}$. The rear-end collision produces a remnant that is greater than the mass of Mimas and can be excluded as a plausible scenario.

velocity to explore the disruption process for comparison to hydrodynamics calculations. During the impact and disruption, the particle collisions are elastic and non-dissipative with a coefficient of restitution $\epsilon=1$. We are in effect using the rubble-pile code as a proxy to model a nearly incompressible dense fluid following a hard-sphere EOS rather than an actual collection of boulders bouncing off of each other at low relative velocities in a non-destructive but dissipative manner. For $\epsilon \approx 0.1-0.9$, as is often used in simulations of asteroid collisions, we determine in simulations that hypervelocity impacts dissipate energy at an artificially high rate and effectively quench disruption. For elastic collisions, we find in the end that the total energy of the system is conserved to within $1 \%$ for our choice of timestep. In rubble-pile simulations, the heating manifests itself as an increase in the velocity dispersion and decrease in particle number density as the hard-sphere "gas" absorbs the energy. After the disruption and dispersal of the mass along a ring, we use a coefficient of restitution $\epsilon<1$ to follow the dissipative evolution of the ring over 30 orbits when relative collision velocities are much smaller.

From the scaling relation of Movshovitz et al. (2015), one expects $Q_{D}^{*}=1.36 \times 10^{5} \mathrm{~J} / \mathrm{kg}$ for the ring parent moon model. We used 5 relative velocities between $11-44 \mathrm{~km} / \mathrm{s}$ in steps of $2^{1 / 2} \times$ and 4 comet masses ranging from $0.94-7.5 \times 10^{16} \mathrm{~kg}$ in steps of $2 \times$ corresponding to impact energies per unit mass ranging from $Q_{D}=0.015-$ $3.8 \times 10^{6} \mathrm{~J} / \mathrm{kg}$. In each case, we followed the collision well past the impact and partial unbinding of mass to the re-accretion of material into a bound body. We estimated the mass of the bound remnant using a friends-of-friends particle grouping method similar to algorithms used for finding halos in cosmological simulations. Figure 3 plots the collision energy per unit mass $Q_{D}$ versus the remnant bound mass for the different comet:moon mass ratios. The plot shows a grouping of simulations near the predicted value of $Q_{D}^{*}=1.36 \times 10^{5} \mathrm{~J} / \mathrm{kg}$ and a remnant mass of $50 \%$ of the initial mass. The implication is that rubble pile simulations with hypervelocity impacts reproduce the results of hydrodynamic simulations of disruption. More complex hydrodynamic simulations should be done eventually to validate these findings but this set of experiments gives us confidence that the use of rubble piles in this context is a reasonable approximation.

\section{Ring parent moon disruption in orbit about Saturn}

We now proceed to simulate the disruption of the ring parent moon modeled as a Roche ellipsoid in orbit about Saturn. Saturn's gravitational field is modeled as a background potential within the N-body code. The potential is represented as a spherical harmonic expansion using Saturn's mass and zonal harmonics from Table 3 of Jacobson et al. (2006) to quadrupole order. We examine three representative collisional scenarios where the comet impacts the moon within the orbital plane from the rear-end (trailing face), side-on and head-on (leading face) directions corresponding to different relative velocities and impact energies (Table 27. Figure 4 and the accompanying animation present the result of the head-on simulation and illustrate the disruption after impact and the ring formation process.

In all cases, the comet is obliterated on impact and deposits its energy in a point-like explosion on the surface of the target moon. The impact ejecta escapes the moon and spreads out along the orbit (Figure 5). In the head-on collision, the moon is almost completely disrupted and the impact ejecta leads the orbit ending up on eccentric trajectories with apogees larger than the initial orbital radius. After one orbit, only 8\% of the mass remains in a bound object composed of about half of the original rocky core with all of the icy mantle liberated to form a ring (Fig. 6).

In the side-on and rear-end collisions, a significant fraction of the icy mantle becomes unbound but the rocky core remains intact with a depleted icy mantle in remnants which contain a mass of $37 \%$ and $51 \%$ of the original moon respectively ( 0.74 and $1.02 \times$ the mass of Mimas). The remnant bound mass depends on the impact energy as shown 


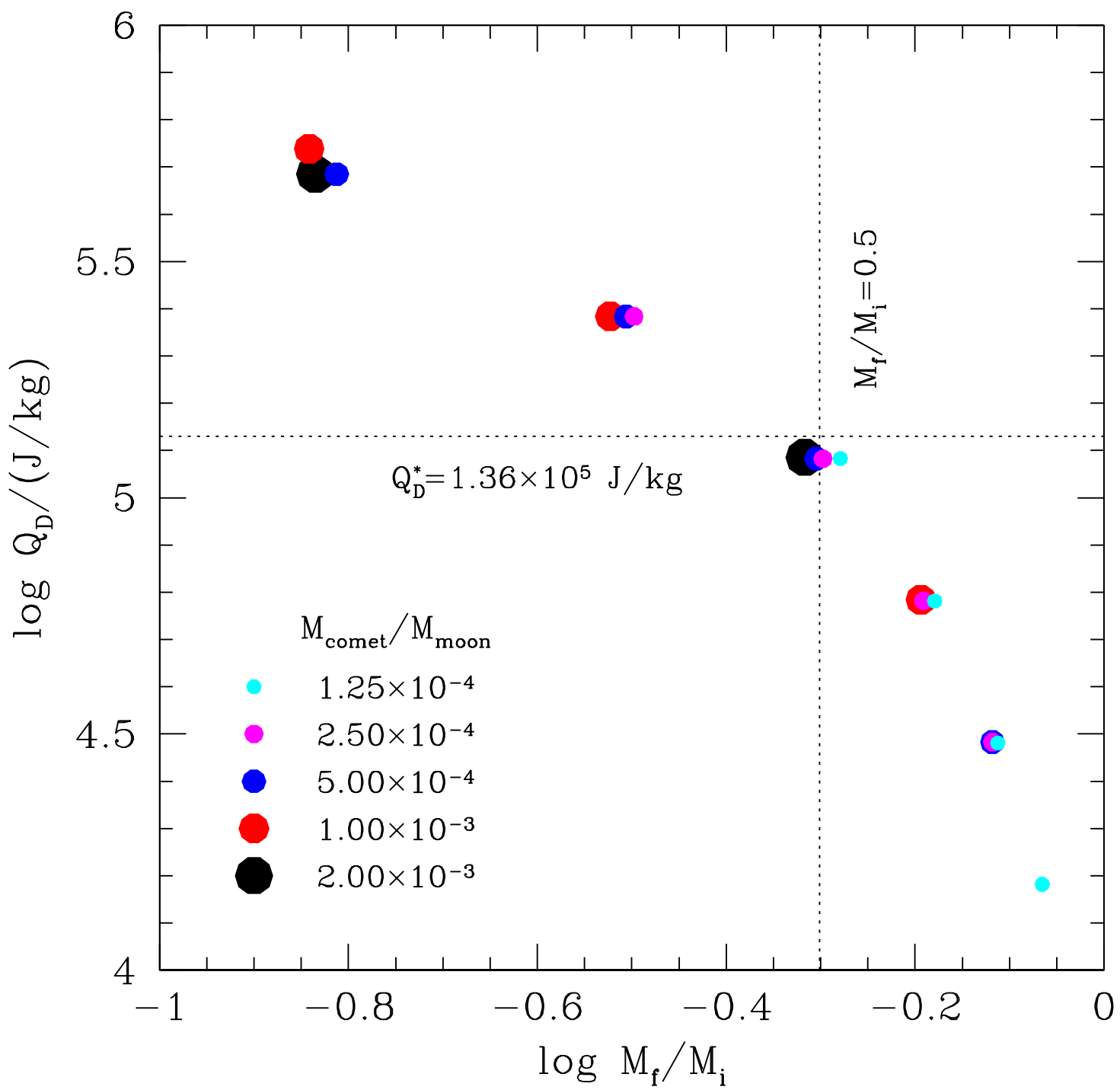

Figure 3: The impact energy per unit mass versus the remnant bound mass for a range of hypervelocity impacts on the putative ring parent moon model. Both the mass and relative velocity of the impactor are varied to explore the behavior of the collisional disruption process. The groups of points at different impact energies reveal that the controlling factor in collisional disruption is the energy per unit mass. The impactor to target mass ratio is less important as long as the ratio is small. The plot also reveals that the critical disruption energy $Q_{D}^{*}$ for the ring parent moon agrees with the scaling relation of Movshovitz et al. (2015). 


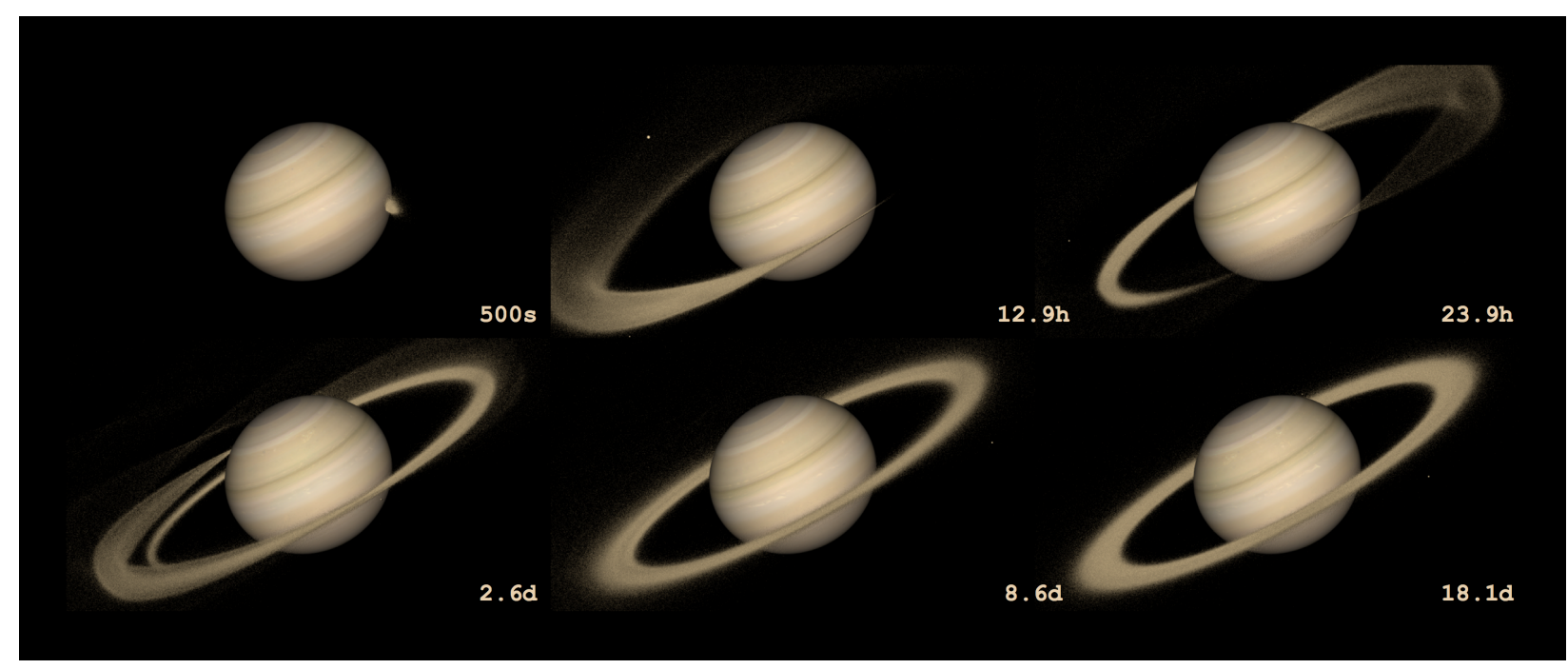

Figure 4: The formation of a ring around Saturn from the disruption of a parent icy moon after a collision with a comet. The moon is initially on a circular orbit with radius $a=140000 \mathrm{~km}$ with an orbital period of $14.9 \mathrm{~h}$. The moon has a mass of $M=7.5 \times 10^{19} \mathrm{~kg}$ and is differentiated with an icy mantle containing $84 \%$ of the mass and a rocky core containing the remaining mass. In this example, a comet of mass $M_{\text {comet }}=3.75 \times 10^{16} \mathrm{~kg}$ collides with the moon head-on within the orbital plane with relative velocity $v_{r e l}=44 \mathrm{~km} / \mathrm{s}$ and disrupts the moon leaving a remnant rocky core containing $8 \%$ of the original mass. The debris spreads along the orbital radius with inelastic collisions between particles leading to a thin ring in Saturn's equatorial plane within a few weeks. At the end of the simulation, the ring is composed of $91 \%$ ice with a radial width of approximately $10,000 \mathrm{~km}$. See accompanying animation at this link: https://youtu.be/UtVnftTd1tA

in collisional disruption studies (Movshovitz et al. 2015) though it seems for the lowest energy impact corresponding to the rear-end collision significantly more mass is lost compared with isolated targets. In the case of a moon near the Roche radius in orbit around Saturn, more mass can become unbound for a given impact energy than after the collision because of the small size of the Hill sphere. In these simulations, the Hill radius of the parent moon is only $700 \mathrm{~km}$ - just a few times the moon's radius. Debris that would normally remained bound to a disrupted moon and re-accrete can instead escape when it moves beyond the Hill radius and mix with the forming ring system. Lower energy impacts can therefore still be effective at unbinding a large fraction of the mass and forming a ring.

After the moon disrupts, the debris settles along the orbit to form an initial squashed toroidal distribution of particles with a radial width $\sim 10000 \mathrm{~km}$ (full-width half maximum of the surface density) and a RMS scale-height of $\sim 1000 \mathrm{~km}$. The evolution of this system is followed for 30 orbits. Once the debris disperses along the orbit, we allow the collisions to be inelastic using coefficient of restitution $\epsilon=0.9$ with a slight modification to permit elastic collisions with $\epsilon=1.0$ for relative velocities with $v<50 \mathrm{~m} / \mathrm{s}$ to prevent runaway clumping of particles (see Appendix A). This value of $\epsilon$ is in accord with laboratory measurements of low velocity impacts of ice spheres (Higa et al. (1996). To save computation time, the remnant moon is extracted after one orbit and replaced with a single sink particle moving along the same trajectory. Particles that collide with this sink particle are removed from the simulation and their mass and momentum are absorbed. The initial velocity dispersion is $\sigma \sim 0.5 \mathrm{~km} / \mathrm{s}$ so collisions of particles in the forming rings are energetic enough to initiate a collisional cascade (Dohnanyi, 1969) that will break up the particles into smaller pieces as they dissipate energy inelastically. Collisions of ice projectiles in experiments with relative velocities $>10 \mathrm{~m} / \mathrm{s}$ are expected to shatter (Hartmann, 1978). The number of particles should grow and lead to an increase in the ring optical depth and collision rate until most of the initial random kinetic energy from the disruption is dissipated in inelastic collisions.

It is not possible to simulate a collisional cascade with the existing rubble pile code so instead we use an ad hoc method to approximate the expected growing optical depth $\tau=N \pi r_{p}^{2}$ where $N$ is the particle surface number density and $r_{p}$ is the simulation particle radius. We increase the optical depth of the forming rings artificially by growing the cross sectional area (increasing the radius) of the particles in two stages after the debris settles into a ring. In this way, the simulation particles are transformed into scaled-particles to increase the ring optical depth and collision rate using a fixed number of particles (Rein et al., 2010). As the ring evolves and spreads radially, the optical depth drops. 


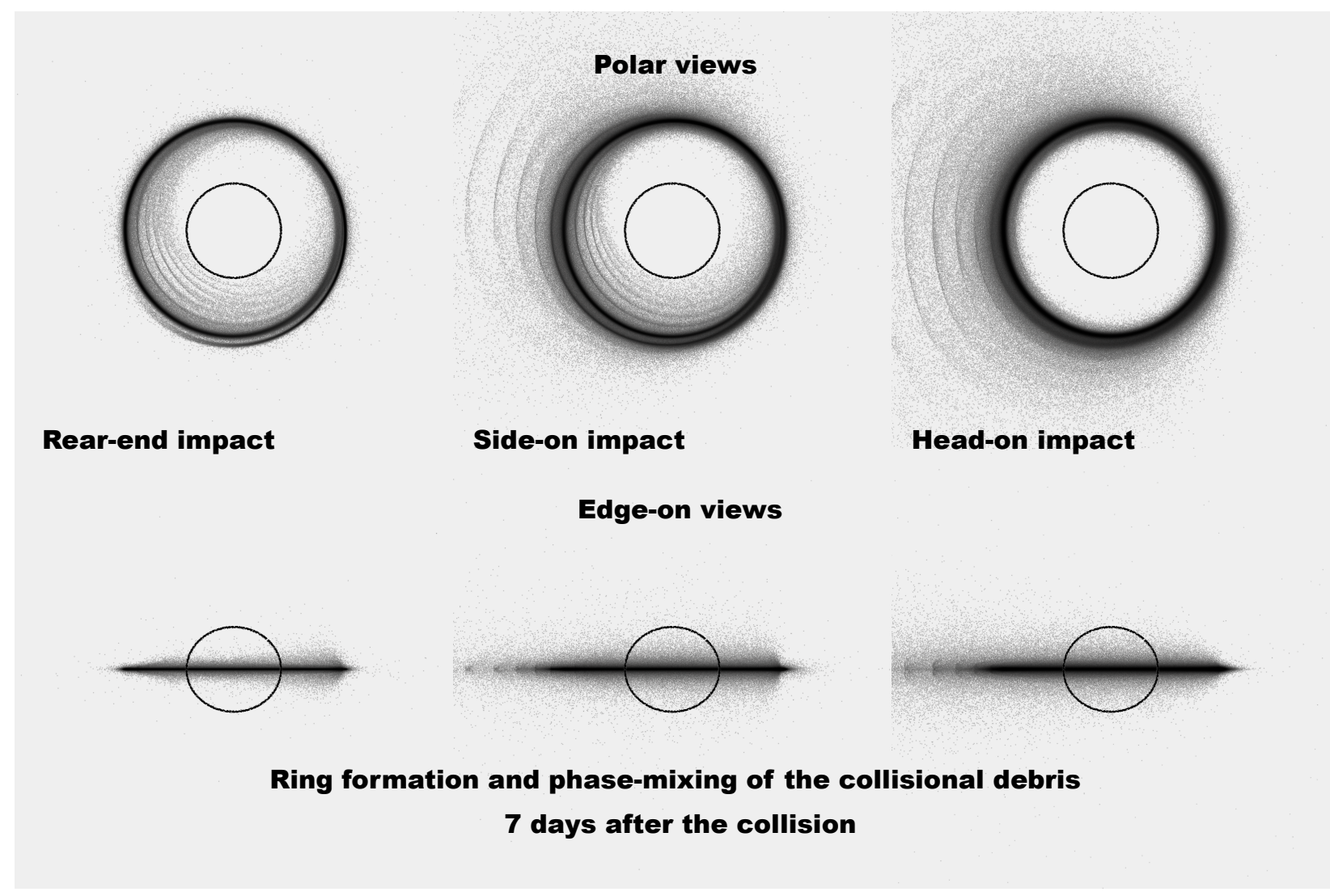

Figure 5: The distribution of collisional debris and the formation of a ring in the three scenarios where the comet impactor collides with the ring parent moon from the rear, the side and head on. The comet orbit is coplanar with the target moon. The polar and edge-on views of the debris are shown 7 days or approximately 11 orbits after the impact. The central circle or ellipse represents Saturn to scale. In the rear-end collision, the impact ejecta trails the orbit and falls into Saturn and phase mixes. Particles pile up on orbital turning points leading to grooves in the distribution. For the side-on collision, debris trails and leads the orbit leading to grooves inside and outside of the orbit. In the head-on collision, the debris leads the orbit and the grooves are outside the orbit. Over time, the groove features are erased as the particles on eccentric orbits circularize after successive collisions with the forming ring. The simulations end after about 20 days or 30 orbits and a broad featureless ring develops by this time. See the animation of this process at this link: https://youtu.be/t8GtvzyD0xw

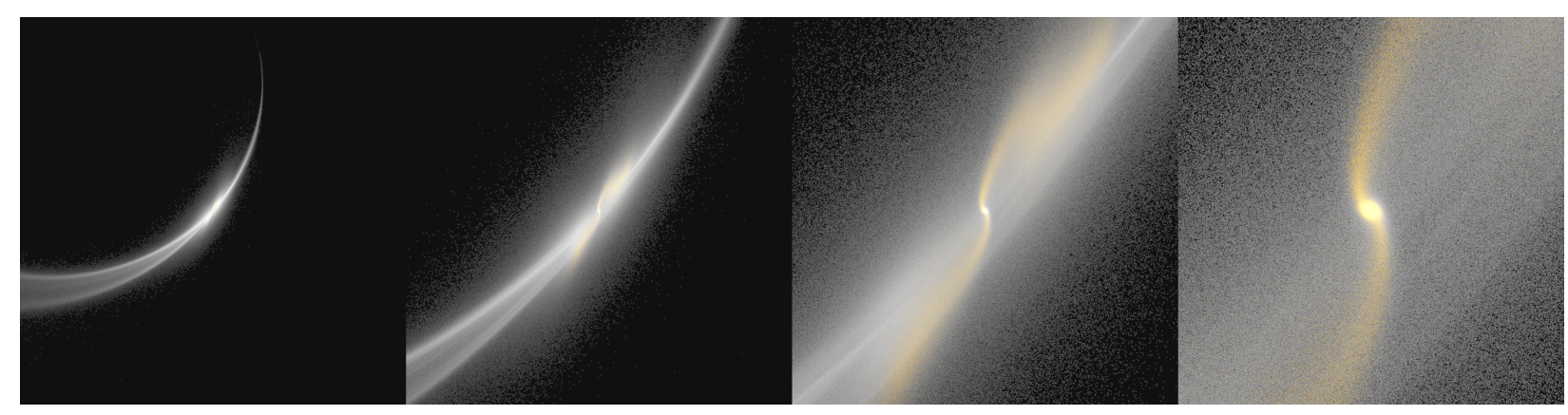

Figure 6: Zooming in on the collisional remnant in the head-on collision simulation one orbit after the impact. Ice particles are rendered in white and rock in red. The width of the images from left to right are $288000,72000,18000$, and $4500 \mathrm{~km}$. The red central spheroid of the remnant is approximately $300 \mathrm{~km}$ across. This simulation is the most energetic of the three and almost disrupts the moon entirely. All of the icy mantle of the ring parent moon is ejected while leaving behind the rocky core. Rock debris leaks away from the Roche lobes of the nearly disrupted moon and mixes with the forming ring leading to an ice fraction of $91 \%$ consistent with the inferred composition of the ring. 
When $\tau \approx 0.1$, the particle cross sectional areas are enlarged $10 \times$ to bring the optical depth back to $\tau \approx 1$. This greater collision rate emulates the effect of a collisional cascade and speeds up the dissipation of energy and relaxation of the ring. The dissipation of energy from inelastic collisions between the particles cause the ring to spread radially and become vertically thinner (Brahic, 1977). The ring particle orbits also undergo orbital phase mixing spreading azimuthally to form a ring with an approximate Gaussian surface density profile (Figure 5). Given the idealized nature of these calculations, the appropriate value for $\epsilon$ is uncertain and the chosen rate of growth of the ring optical depth to emulate the effect of a collisional cascade is also not well-defined. At the end of these simulations, the velocity dispersions are still relatively high with $\sigma \sim 50 \mathrm{~m} / \mathrm{s}$ (see below) much larger than the current value of $\sigma \sim 0.01 ; \mathrm{m} / \mathrm{s}$ (Goldreich and Tremaine, 1978). Despite the uncertainties, the rate of dissipation in the nascent ring in this early stage may be over-estimated but one expects the structural evolution to proceed in qualitatively the same way since angular momentum is conserved.

Figure 7 shows the surface density profile, scale height and velocity dispersion of the ring at three times for the head-on collision and reveals the rapid transformation of the spreading debris cloud from a point-like collisional disruption into a ring system. The final state of these simulations can be thought of the initial conditions for the subsequent long term phase of viscous spreading of the rings into their current state as described by (Salmon et al., 2010). The three simulations span a range of impact energies and each form a ring system with a high ice fraction (see Table 2). For lower impact energies, a significant bound mass made predominantly of the original rocky core can survive while the debris is composed of the icy mantle. This is a simple way to explain the icy purity of the rings though it requires an initial moon that is differentiated with a relatively high ice fraction of $84 \%$ - albeit in the expected range for Saturn's moons. Over the long term, the remnant should accrete a significant fraction of the nascent ring as it spreads viscously. The least energetic rear-end collision produces a remnant that is greater than the mass of Mimas and so can be excluded as a possibility. The other two models have remnants with less mass than Mimas that will grow in size by accretion of the spreading debris. A more thorough study involving moons with different ice fractions and cometary impact energies can set stronger constraints on the range of plausible scenarios once the mass of the rings are determined from the Cassini ring flyby data. The 3 simulations presented here demonstrate that it should be relatively easy to find simulation parameters that preserve a rocky core while liberating an icy mantle.

The remnant acts as a new seed for the re-accretion of debris to form a new moon which one expects to clear out the debris within its orbital radius within a timescale as short as $10^{3}$ years (Crida and Charnoz, 2012). The long term evolution of a collision remnant embedded within a newly formed ring should be followed to find scenarios that will produce a Mimas of the right size while some of the debris moves into the Roche zone. One expects the newly formed Mimas to experience resonant interactions with the particles in the spreading ring. The resulting torques lead to outward migration with the orbital radius $a$ reaching its current value with a timescale:

$$
\tau_{\text {migration }}=\left(\frac{1}{a} \frac{d a}{d t}\right)^{-1}=0.60 \frac{M_{\text {Saturn }}^{2}}{a^{2} \Sigma \Omega M_{\text {Mimas }}}\left|\frac{a-r}{a}\right|^{3}
$$

where $\Sigma$ is the surface density of the ring, $\Omega$ is the orbital frequency and $r$ is the radius of the outer edge of the ring (Goldreich and Tremaine, 1982). From these simulations, the initial central surface density after settling down into a thin ring is $\Sigma \approx 5000 \mathrm{~kg} / \mathrm{m}^{2}$. With the ring's outer edge at $r=140000 ; \mathrm{km}$ and the current radius of Mimas' orbit at $a=185400 ; \mathrm{km}$ lead to a migration timescale of $\tau_{\text {migration }}=120$ Myrs. In the region between the rings and Mimas, torques from ring interactions are greater than the torques from tidal interactions with Saturn (Crida and Charnoz, 2012). Since the mass of the ring and Mimas are comparable, the outward migration of Mimas will result in a back reaction from the ring which will cause it to spread inward more rapidly than expected from collisional viscous effects alone and its mean surface density will decline as the ring spreads to the current estimated value of $\Sigma=1000 \mathrm{~kg} / \mathrm{m}^{2}$ for the $\mathrm{B}$ ring. This sets a more conservative timescale of a few times $\tau_{\text {migration }}$ because of the inverse dependence on $\Sigma$. The recent value from Lainey et al. (2017) for Saturn's tidal dissipation factor $k_{2, S} / Q_{S} \approx 1.6 \times 10^{-4}$ predict a tidal migration time of a Mimas from $a=140000 \mathrm{~km}$ to its current orbital radius in only 450 Myr. More detailed dynamical calculations of this system are required but the timescale for moving Mimas out from the edge of the rings to its current position in a few hundred million years consistent with the assumption of a recent formation time for the rings. 

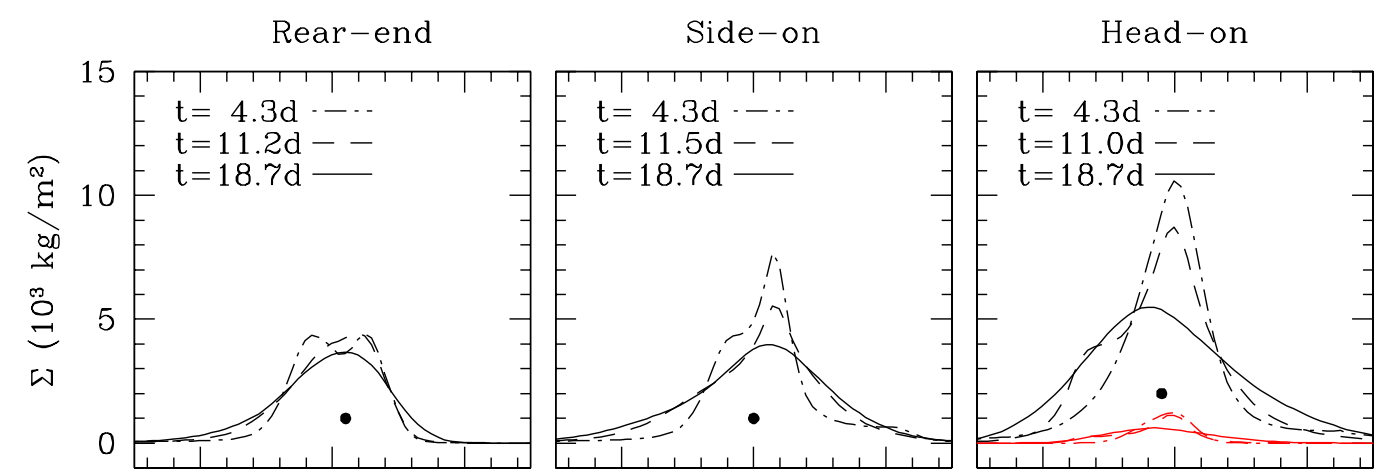

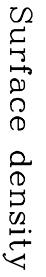
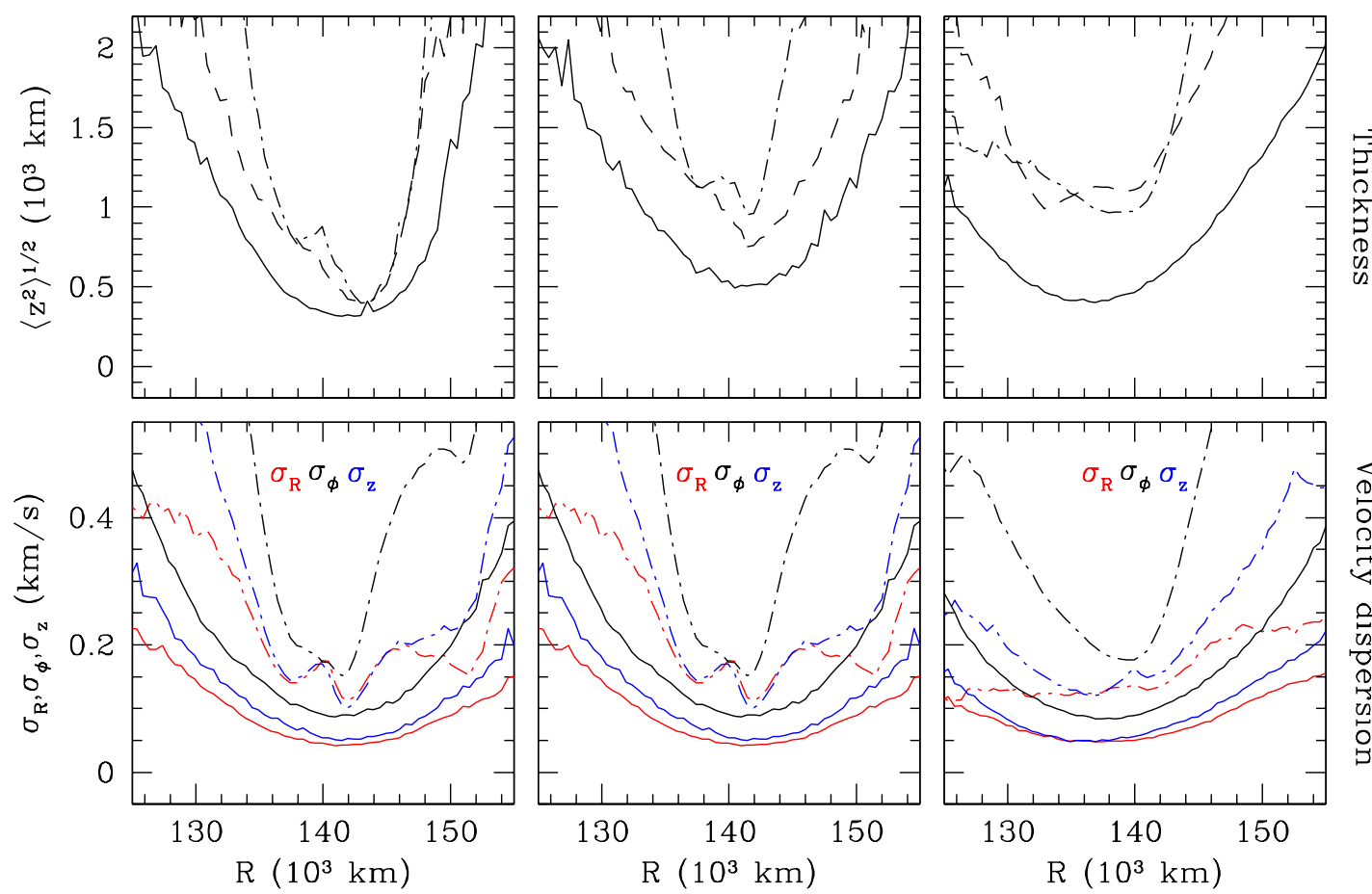

0
0
0
0
0
4
8
0
0
0
0
0
0.
0
0
0

Figure 7: Structure and kinematics of the ring for the three collision cases: rear-end, side-on and head-on at three times during the formation and evolution of the ring. The structure is measured from the azimuthally averaged surface density and the thickness as measured by the RMS of the particles vertical position with respect to the equatorial plane. The kinematics are described by the azimuthally averaged velocity ellipsoid in different radial bins. Within a few weeks, the ring surface density has a Gaussian shape with maximum centered on the initial orbital radius and a full width half maximum of about $10000 \mathrm{~km}$. Collisional dissipation leads to the circularization of the ring particle orbits and the decline in velocity dispersion to $0.05-0.1 \mathrm{~km} / \mathrm{s}$. In each case, the disruption is not complete and a remnant remains in orbit embedded within the debris - the radial position of the remnant's semi-major axis is shown by the black dot. For the rear-end and side-on collisions, the remnant contains nearly half of the original rocky core and the ring is pure ice. For the head-on collision, the disruption is nearly complete but a remnant core containing half the rock remains with the debris (shown in red in the surface density plot) mixing with the ring. 


\section{Discussion}

The mechanism responsible for the creation of Saturn's rings is intertwined with the origin and subsequent dynamical evolution of Saturn's icy mid-sized moons. In this section, we discuss some of the implications for the Saturn system from the proposed collisional disruption mechanism.

\subsection{The age of Mimas}

The scenario proposed in this paper suggests a coeval origin of Saturn's rings and Mimas within the past few hundred million years. This is contrary to the conventional view that Mimas formed primordially with Saturn and the other inner mid-sized icy moons more than 4 billion years ago. The heavily cratered surface of Mimas is consistent with a very old age based on measurements of the size distribution of craters if they originate from objects on heliocentric orbits (Zahnle et al., 2003). However, measurements of the libration of Mimas by the Cassini images (Tajeddine et al., 2014) are at odds with a primordial origin. If Mimas formed primordially, one expects it to be either a homogeneous or differentiated body in hydrostatic equilibrium but direct measurement of libration show anomalies inconsistent with these states. Two explanations that can explain the anomaly are 1) an internal ocean or 2) a non-hydrostatic ellipsoidal rocky core. We have shown that an icy ring can form from the partial disruption of a differentiated ring parent moon leaving behind a rocky core that subsequently re-accretes to become Mimas while being pushed out to its current position through a combination of resonant interactions with the ring and tidal dissipation. It seems plausible that the post-impact disturbed remnant rocky core would not have time to relax to hydrostatic equilibrium and so support this hypothesis as the origin for the anomalous libration. Recent models of the thermal, structural and orbital evolution of Mimas are consistent with a late, layered formation scenario similar to the one proposed here (Neveu and Rhoden, 2017).

\subsection{Mean motion resonances and the heating of Enceladus}

The ring forming scenario has a further implication that may help in understanding another puzzle in Saturn's system of moons: the heat source of Enceladus endogenic activity. Spencer et al. (2006) have measured the current heat output from Enceladus as $5.8 \pm 1.9 \mathrm{GW}$ which is much larger than the heat input expected from either radiogenic sources in the rock or tidal heating from the current MMR with Dione. If a ring parent moon is in MMR with Dione and Enceladus prior to disruption, one expects the transfer of angular momentum outward from tidal friction to excite a larger eccentricity in the orbit of Enceladus which in turn increases the rate of tidal heating (Squyres et al., 1983, Peale, 1999). It has been argued before that the outward transfer of angular momentum from a possible past orbital resonance with Janus may have pumped up the eccentricity of Enceladus thus providing a heat source (Lissauer et al., 1984). The mass of Janus proved to be too small for this heating mechanism to work but the ring parent moon proposed here may have enough mass to increase the tidal heating rate to values significantly above the current rate.

To illustrate this, we assume that the ring parent moon, Enceladus and Dione are in MMR prior to the comet collision with orbital frequencies in the ratio of 4:2:1. While in resonance, tidal friction will cause the moons to migrate outwards in tandem while maintaining the ratio of orbital frequencies and semi-major axes. For 3 moons in MMR, the time for the innermost moon to migrate from semi-major axis $a_{i}$ to $a_{f}$ is given by:

$$
t=t_{\text {migrate }}\left[1-\left(\frac{a_{i}}{a_{f}}\right)^{13 / 2}\right]
$$

where the time constant is:

$$
t_{\text {migrate }}=\left[\frac{39}{2} \frac{m_{1}}{M_{S}}\left(\frac{R_{S}}{a_{1}}\right)^{5} \frac{k_{2, S}}{Q_{S}} n_{1} \eta\right]^{-1},
$$

with

$$
\eta=\frac{1+\left(m_{2} / m_{1}\right)^{2}\left(a_{2} / a_{1}\right)^{-6}+\left(m_{3} / m_{1}\right)^{2}\left(a_{3} / a_{1}\right)^{-6}}{1+\left(m_{2} / m_{1}\right)\left(a_{2} / a_{1}\right)^{1 / 2}+\left(m_{3} / m_{1}\right)\left(a_{3} / a_{1}\right)^{1 / 2}}
$$

and $m_{1}, m_{2}, m_{3}$ are the masses of the moons, $a_{1}, a_{2}$, and $a_{3}$ are semi-major axes of the moons' orbits, $n_{1}$ is the orbital frequency of the innermost moon, $R_{S}$ and $M_{S}$ are the radius and mass of Saturn and $k_{2, S}$ and $Q_{S}$ are Saturn's Love number and dissipation function (e.g., Murray and Dermott |2000). The conventional value for Saturn's dissipation 
term is $k_{2, S} / Q_{S}=2 \times 10^{-5}$ calculated under the assumption that Mimas migrates from the synchronous radius to its current radius over 4.6 Gyr (the age of the solar system) by tidal evolution (Goldreich and Soter, 1966). With this value, the time for the ring parent moon considered here in MMR to migrate from an orbital radius of $a=130000$ $\mathrm{km}$ to $150000 \mathrm{~km}$ is $4.5 \mathrm{Gyr}$ showing that it is possible to trap such a moon near the Roche radius for the age of the solar system. For $k_{2, S} / Q_{S}=1.6 \times 10^{-4}$ proposed by Lainey et al. (2017), this timescale is only 0.6 Gyr. However, Lainey's value for Saturn's dissipation term implies a recent origin for most of Saturn's icy moons, since all moons out to Dione's orbital radius would migrate to their current locations in less than 2 Gyr (Ćuk et al., 2016).

Assuming nearly circular orbits and conservation of energy and angular momentum, one can also calculate the net heat flow into the moons from orbital binding energy as:

$$
H=\sum_{i} n_{i} T_{i}+\frac{2 \sum_{i} T_{i} \times \sum_{i} E_{i}}{\sum_{i} L_{i}}
$$

where $n_{i}$ are the satellite orbital frequencies, $T_{i}$ are the torques on the satellites due to tidal evolution, $E_{i}$ and $L_{i}$ are the satellite orbital binding energies and angular momenta (Lissauer et al., 1984, Meyer and Wisdom, 2007). The torque from tidal evolution is given by:

$$
T=\frac{3}{2} \frac{G M_{m}^{2} R_{S}^{5}}{a^{6}} \frac{k_{2 S}}{Q_{S}}
$$

where $M_{m}$ is the mass of the moon, $a$ is the orbital radius and $R_{S}$ is the radius of Saturn. A system of satellites in MMR will excite orbital eccentricities in the moons with equilibrium values that balance the heat gain from the tidal evolution of the orbits and the heat loss from internal dissipation in the moons caused by the varying tidal field on the eccentric orbit. The dissipation rate for a synchronously rotating moon is:

$$
\frac{d E}{d t}=\frac{21}{2} \frac{k_{2, m}}{Q_{m}} \frac{G M_{S}^{2} n R_{m}^{5}}{a^{6}} e^{2}
$$

where properties of the moon are denoted with the subscript $m$ and $e$ is the orbital eccentricity assumed to be small (Peale, 1999). (For the more general formulae, see Wisdom (2008) - for $e=0.1$ the dissipation rate is about $20 \%$ larger than that given by Eq. 11. The satellite Love number can be estimated by:

$$
k_{2, m}=\frac{3 / 2}{1+19 \mu /(2 \rho g R)}
$$

with rigidity $\mu=4 \times 10^{9} \mathrm{Nm}^{-2}$ and $\rho$ is the density, $g$ is the surface acceleration and $R$ is the radius with the satellite dissipation term in the range $Q_{m}=20-100$ (Meyer and Wisdom, 2007).

For Enceladus and Dione in a 2:1 resonance, the above analysis was used to estimate a heating rate of $1.1 \mathrm{GW}$ (Meyer and Wisdom, 2007) which is much less than the measured heat output of Enceladus (Spencer et al., 2006). If a ring parent moon existed in the past, this heating rate would be significantly larger. Let us consider a parent moon that is twice the mass of Mimas $M=7.5 \times 10^{19} \mathrm{~kg}$ used in our simulation that begins at an orbital radius of $a=135000 \mathrm{~km}$ just beyond the Roche radius with Enceladus and Dione in MMR with orbital frequencies in the ratio 4:2:1. Using the above equations with $Q_{m}=20$ for the moons, one finds a heat flow from equation 9 into the 3 moons of $H=107 \mathrm{GW}$ (and $H \approx 1000 \mathrm{GW}$ for Lainey's $Q_{S}$ !). This is 2 orders of magnitude larger than the current tidal heating rate estimated above. Prior to the ring parent moon's destruction, this higher heating rate on Enceladus implies that its icy fraction may have been completely molten creating a smooth crater-free surface (Smith et al., 1982). After the disruption of the parent moon and the formation of the ring, the eccentricity of Enceladus would decay to its current value with a time constant of order $10^{8}$ years (Meyer and Wisdom, 2007) with the heating rate falling off and Enceladus refreezing. It has been argued that a previous molten state driven by a more eccentric orbit in the past is needed to explain the observed heat output under the more modest tidal heating rate existing today (Ojakangas and Stevenson, 1986).

To further understand this scenario, we carried out orbital integrations of an idealized co-planar system containing a ring parent moon, Enceladus and Dione in MMR to illustrate both the long term stability of this system and heating rates. We modified the Mercury N-body code (Chambers, 1999) to include velocity-dependent fictitious forces to mimic tidal evolution for the moons (e.g., Lee and Peale, 2002). Torques from tidal evolution and internal dissipation 
are included allowing an exploration of the evolution of the semi-major axis, eccentricity and tidal heating of the satellites. For Saturn, we use both the conventional value of $k_{2, S} / Q_{S}=2 \times 10^{-5}$ for the tidal dissipation term as well as the recent Lainey et al. (2017) value of $k_{2, S} / Q_{S}=1.6 \times 10^{-4}$. We compute the satellite Love numbers from equation 12 with different values of tidal dissipation $Q_{m}$ for the moons. Since tidal migration is very slow compared to the orbital period, we speed up the calculations by increasing the $Q$ factors by $100 \times$ (e.g., Meyer and Wisdom, 2008) and rescaling the timescale in the plots (Meyer and Wisdom, 2008) (Note: calculations with a speed up of 1000× also gave the same result). We begin the integration with the parent moon on a nominally circular co-planar orbit with $a=135000 \mathrm{~km}$ and Enceladus and Dione with orbital radii slightly larger than their $2: 1$ and $4: 1$ resonant positions with respect to this initial radius.

Figures 8 and 9 show the evolution of the semi-major axes and eccentricities of the 3 moon system with a ring parent moon of twice the mass of Mimas as assumed in the disruption simulations with the conventional value for Saturn's tidal dissipation. Initially the semi-major axis of the ring parent grows rapidly in response to tidal friction with Saturn but the 3 moons quickly lock into MMR slowing down the ring parent's outward migration significantly. The ring parent moon can therefore stay near the Roche zone for the age of the solar system and the 3 moon system is stable for at least $0.5 \mathrm{Gyr}$ with the conventional value of Saturn's tidal dissipation. The resonance excites eccentricities in the 3 moons and equilibrium values for $e=0.005,0.08$, and 0.02 are reached for the ring parent, Enceladus and Dione respectively leading to heating rates from equation 11 (in the same order) of $H=5,64,37 \mathrm{GW}$. The total heating rate is slightly larger than the value estimated from equation 9 because of the significant eccentricities excited in the moons. For the smaller ring parent moon, the heating rate is sufficient to melt the ice and lead to a differentiated moon as assumed in the model. The heating rate for Enceladus is very high by comparison and implies the existence of an oceanic mantle and volcanically active past. One can imagine significant mass loss during this phase which is consistent with the higher mean density of $\rho=1.65 \mathrm{~g} / \mathrm{cm}^{3}$ measured for Enceladus. Even Dione with 10 times the mass of Enceladus would experience significant heating under these circumstances implying a large subsurface ocean in the past and perhaps more surface geological activity than one might expect from the current state.

We repeated the calculations varying the mass of the ring parent moon and satellite Love numbers with Lainey's value for Saturn's tidal dissipation. For the same ring parent mass with $Q_{m}=20$ for the moons, the radial migration rate is 8 times faster as expected but the 3-moon system only stays in MMR for a short time. The failure of systems with larger ring parent moon masses and values of $Q_{m}$ to stay in MMR is probably due to the highly eccentric orbit excited in Enceladus. Given that press reports suggest a smaller ring mass as determined by Cassini, we examined one case where the ring parent moon is only $1.5 \times$ the mass of Mimas. With $Q_{m}=10$, this system remained in MMR for approximately $0.5 \mathrm{Gyr}$ (see Fig. 10 and Fig. 11). The orbital radius of the ring parent grew by $10 \%$ from 135000 $\mathrm{km}$ to about $150000 \mathrm{~km}$ with the eccentricities of Enceladus and Dione excited to $e=0.125$ and 0.035 respectively resulting in significantly higher tidal heating rates. By the end of this time interval, the system falls out of MMR and the ring parent recommences a rapid outward radial migration. Lainey's tidal factor for Saturn implies that the system of icy satellites out to Dione may only have an age $\sim 1$ Gyr. This calculation suggests it may be possible to trap a ring parent satellite with appropriate properties for this timescale. A more thorough search of satellite parameters is needed to constrain this more precisely.

Finally, we examined the orbital evolution of Enceladus and Dione after the disruption of the satellite for the case using the conventional value of tidal dissipation and ignoring ring torques. We simply remove the ring parent moon from the system in MMR with equilibrium eccentricities and continue the orbital integration for the two remaining moons. Figures 12 and 13 show the evolution of the semi-major axes and eccentricities after the impact. The tidal migration of Enceladus and Dione stalls for a few hundred million years but the two moons eventually settle back into a 2:1 MMR. The eccentricities decay with the timescales expected when resonant forcing is removed and a small eccentricity is re-excited in Dione when they get back into resonance. We note that this illustrative example is not identical to the current MMR between Enceladus and Dione which instead excites an eccentricity in Enceladus rather than Dione. There are in fact a multiplet of possible 2:1 resonances that can excite both inclinations and eccentricities of either Enceladus or Dione or both (Sinclair, 1972; Zhang and Nimmo, 2009). The convergent co-planar orbital scenario illustrated here passes through the $e$-Dione resonance first and the moons are apparently captured here contrary to the current state of the system. Zhang and Nimmo (2009) have argued that capture into the $e$-Dione resonance becomes less probable if Dione has a prior free eccentricity greater than a critical value $e \sim 0.002$. A more thorough examination of the orbital evolution in this scenario with varying values of $k_{2} / Q_{m}$ for the moons might help understand different pathways to the current state. In any case, the timescale for the eccentricity of Enceladus to decay to its 


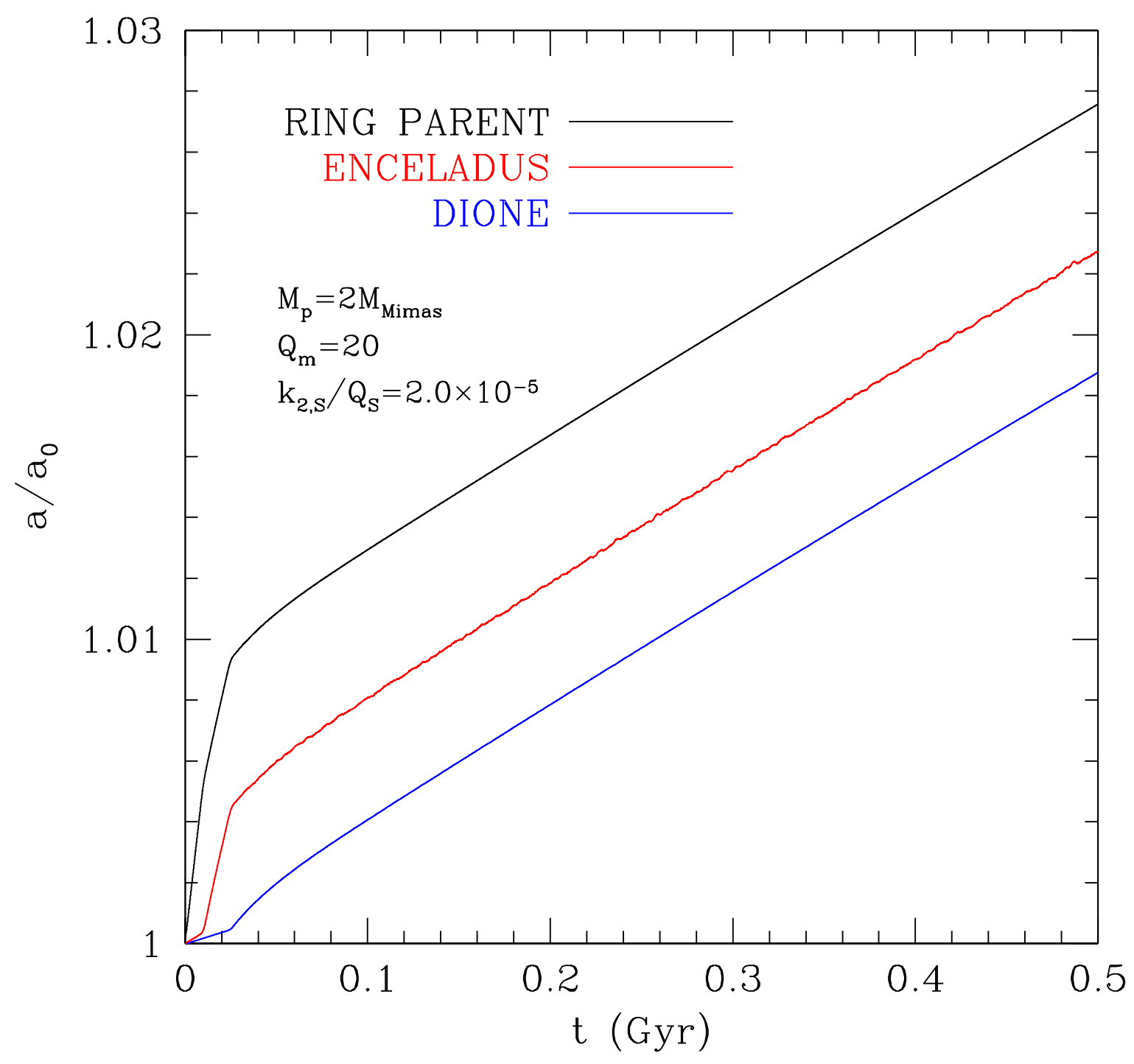

Figure 8: The evolution of the semi-major axes of the ideal co-planar system of 3 moons consisting of the ring parent moon, Enceladus and Dione using a conventional value of Saturn's tidal dissipation term. Tidal dissipation in Saturn causes the semi-major axis to grow. The initial semi-major axes are intentionally set to values slightly out of resonance with values smaller than the current values. The ring parent moon first locks into a 2:1 MMR with Enceladus. This system shortly after enters a MMR with Dione that remains stable to the end of the integration of $0.5 \mathrm{Gyr}$. This calculation suggests that a ring parent moon could be trapped in mean motion resonance near the Roche radius for a long time - perhaps as long as the age of the Solar system. 


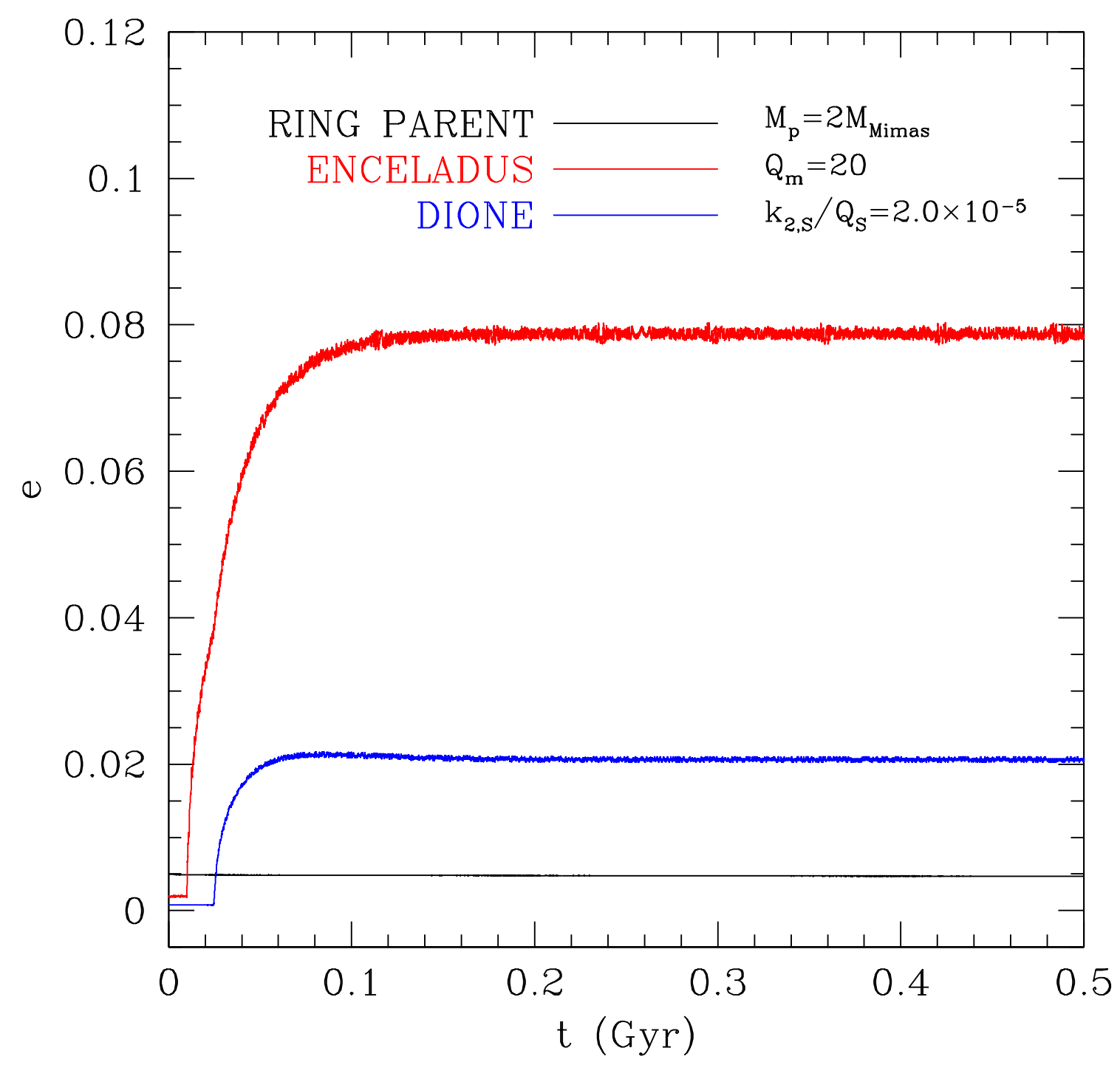

Figure 9: The evolution of the eccentricities of the ideal co-planar system of 3 moons consisting of the ring parent moon, Enceladus and Dione using a conventional value of Saturn's tidal dissipation term. When the moons lock into mean motion resonance, the eccentricities of Enceladus and Dione grow to equilibrium values of $e=0.08$ and $e=0.02$ respectively - values an order of magnitude larger than the current values of $e=0.0047$ and $e=0.0022$. The ring parent's eccentricity settles to $e=0.005$. These equilibrium values depend on the satellite Love numbers and tidal dissipation terms $Q_{m}$. Nevertheless, since the tidal heating is proportional to $e^{2}$ (Peale 1999), we expect internal heating rates about 2 orders of magnitude larger than today in this scenario. Prior to the removal of the ring parent satellite, one therefore expects the icy mantle of Enceladus to be largely molten. 


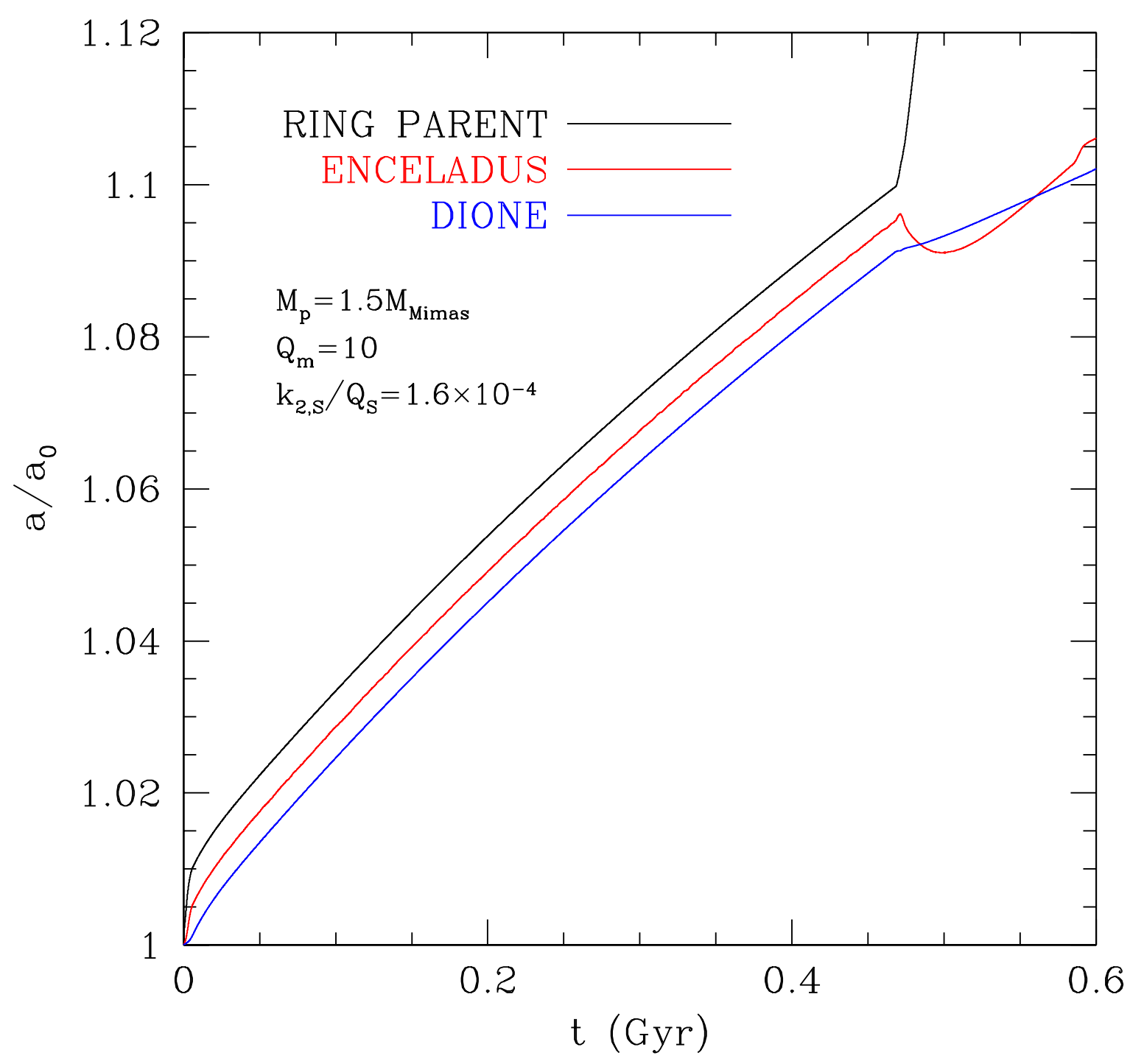

Figure 10: The evolution of the semi-major axes of an ideal system of 3 moons consisting of the ring parent moon, Enceladus and Dione using the Lainey et al. (2017) value of Saturn's tidal dissipation term. The ring parent moon has a mass $M_{p}=1.5 \times M_{\text {Mimas }}$ and Love numbers of the satellites are computed assuming $Q_{m}=10$. This system only stays in MMR for about $0.5 \mathrm{Gyr}$ before exiting the resonant state. 


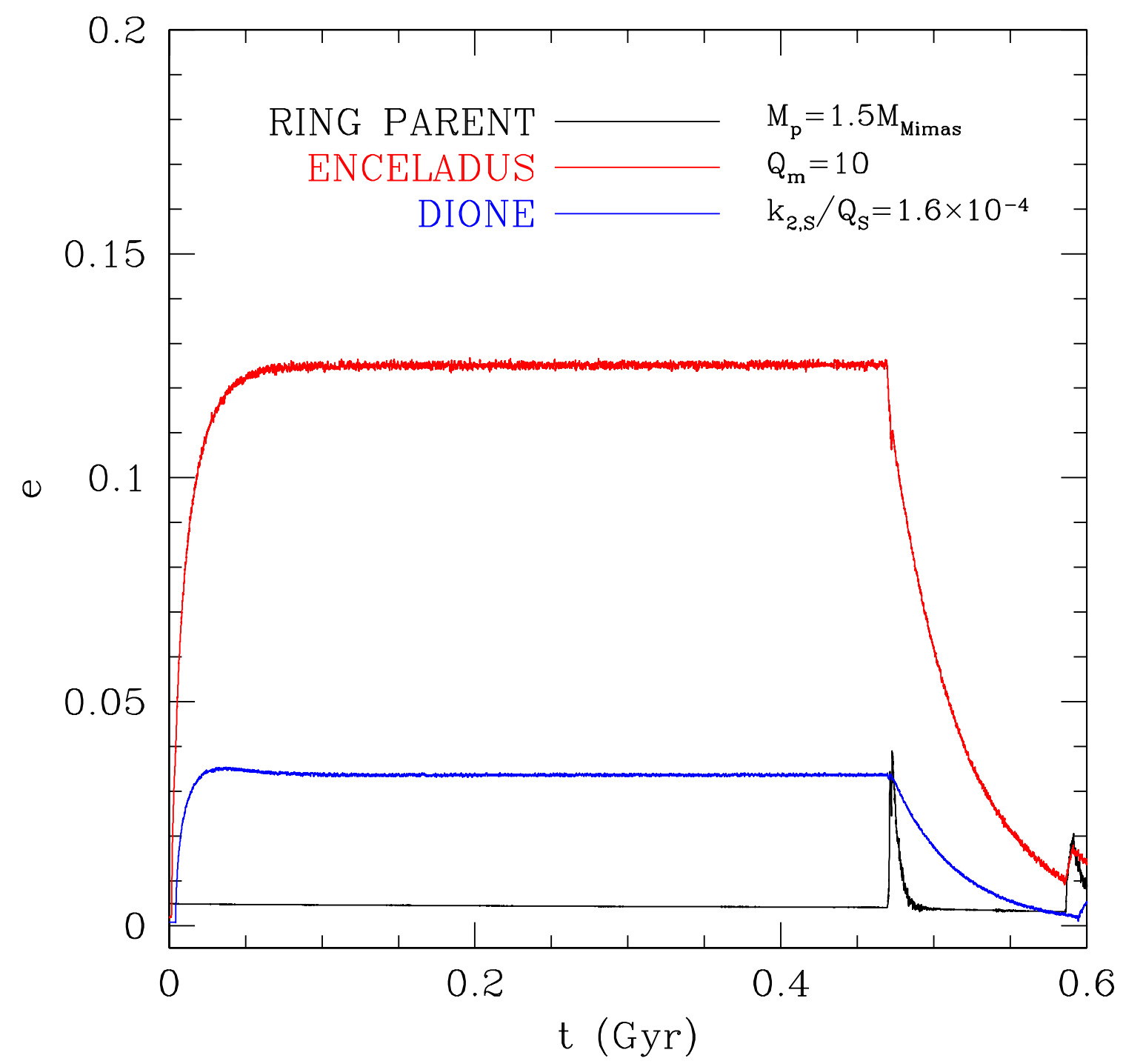

Figure 11: The evolution of the eccentricities of an ideal system of 3 moons consisting of the ring parent moon, Enceladus and Dione using the Lainey et al. (2017) value of Saturn's tidal dissipation term. When the moons lock into MMR, the eccentricities of Enceladus and Dione grow to equilibrium values of $e=0.125$ and $e=0.035$ respectively - values that are larger than the fiducial case using a conventional dissipation term for Saturn. The system abruptly exits MMR around $0.5 \mathrm{Gyr}$ after the start of the integration and the eccentricities decay rapidly. 
present value is approximately $0.3 \mathrm{Gyr}$ which coincides with the expected timescale for the newly formed Mimas to migrate to its orbital radius. One still expects remnant heat from the previous highly eccentric orbit of Enceladus and this may explain why it is still partially molten and quite active despite the low amount of tidal heating at the present time.

\section{Conclusions}

In summary, the collisional disruption mechanism proposed in this paper presents a solution to the origin of Saturn's rings that is consistent with the mass, age and composition of the rings. A differentiated moon with a mass twice that of Mimas and an ice fraction consistent with the other icy moons located near the Roche radius can be disrupted by a heliocentric comet with a radius of $\sim 20 \mathrm{~km}$. Representative simulations show that the icy mantle can be liberated in the collision forming a nearly pure ice ring system with a mass comparable to Mimas while leaving behind a rocky remnant. It is argued that the viscous spreading of the ring of debris will lead to the current ring system and a new satellite composed of the remnant and re-accreted debris that we identify with Mimas. While this event could have occurred any point during the Saturn system's lifetime, the short timescales for viscous spreading of the rings and contamination from meteoroid bombardment suggest that the event happened within the last few hundred million years. The probability that an impact with sufficient destructive energy occurs in this timeframe is $\sim 0.01$ making it a $2-3 \sigma$ event - perhaps unlikely but not wildly so (Zahnle et al., 2003, Dones et al., 2009). The model therefore simultaneously accounts for a mass of the rings comparable to Mimas, a young age and nearly pure ice composition. A further requirement for this model to work is the necessity that the ring parent moon is locked in MMR with Enceladus and Dione so that it remains trapped near the Roche radius prior to destruction. We have demonstrated that this phase would tidally heat Enceladus at a much greater rate than now. Remnant heat might still be present explaining the existence of Enceladus' current heat output, subsurface ocean and surface activity.

During Cassini's Grand Finale tour in 2017, the spacecraft flew close enough to the rings to measure a gravitational perturbation using the radio science experiment and so determine a definitive mass for the rings (Charnoz et al. 2018). The scenario proposed here only works if the ring mass is comparable to the mass of Mimas. If the mass of the rings is close to the upper range of current estimates - about $3 \times$ the mass of Mimas - this scenario seems less likely since it becomes increasingly difficult to keep such a large ring parent moon within the Roche zone because of the stronger torques from tidal friction and the lower probability for disruption by an impact. New as yet unpublished reports suggest that the total mass of rings may be less than half of the mass of Mimas. Once the mass is known precisely, this scenario is strongly constrained and it should be possible to narrow the range of collision energies, impact geometries and the initial composition of the ring parent moon using more sophisticated simulation methods.

\section{Acknowledgments}

I acknowledge Peter Goldreich, Chris Thompson, Yanqin Wu and Luke Dones for discussions that have inspired and clarified points in this paper. I thank referees Luke Dones and Matija Ćuk for comments that pointed to pertinent observations and helped elucidate important physical processes as well as point to many useful references. I also thank Björn Jónsson for permission to use his texture map of Saturn from Cassini data for Figure 4 and the accompanying animation. Simulations were carried out at the computing facilities of the Canadian Institute for Theoretical Astrophysics. This research was financially supported by the Natural Sciences and Engineering Research Council of Canada.

\section{References}

Ballouz, R.L., Richardson, D.C., Michel, P., Schwartz, S.R., Yu, Y., 2015. Numerical simulations of collisional disruption of rotating gravitational aggregates: dependence on material properties. Planetary Space Sci. 107, 29-35. doi 10.1016/j.pss.2014.06.003 arXiv:1409.6650

Barnes, J., Hut, P., 1986. A hierarchical O(N $\log \mathrm{N})$ force-calculation algorithm. Nature 324, 446-449. doi 10.1038/324446a0

Benz, W., Asphaug, E., 1999. Catastrophic disruptions revisited. Icarus 142, 5-20. doi 10.1006/icar.1999.6204 arXiv:astro-ph/9907117 Brahic, A., 1977. Systems of colliding bodies in a gravitational field. I - Numerical simulation of the standard model. Astron. Astrophys. 54, 895-907.

Canup, R.M., 2010. Origin of Saturn's rings and inner moons by mass removal from a lost Titan-sized satellite. Nature 468, $943-946$. doi 10 . 1038 /nature09661 


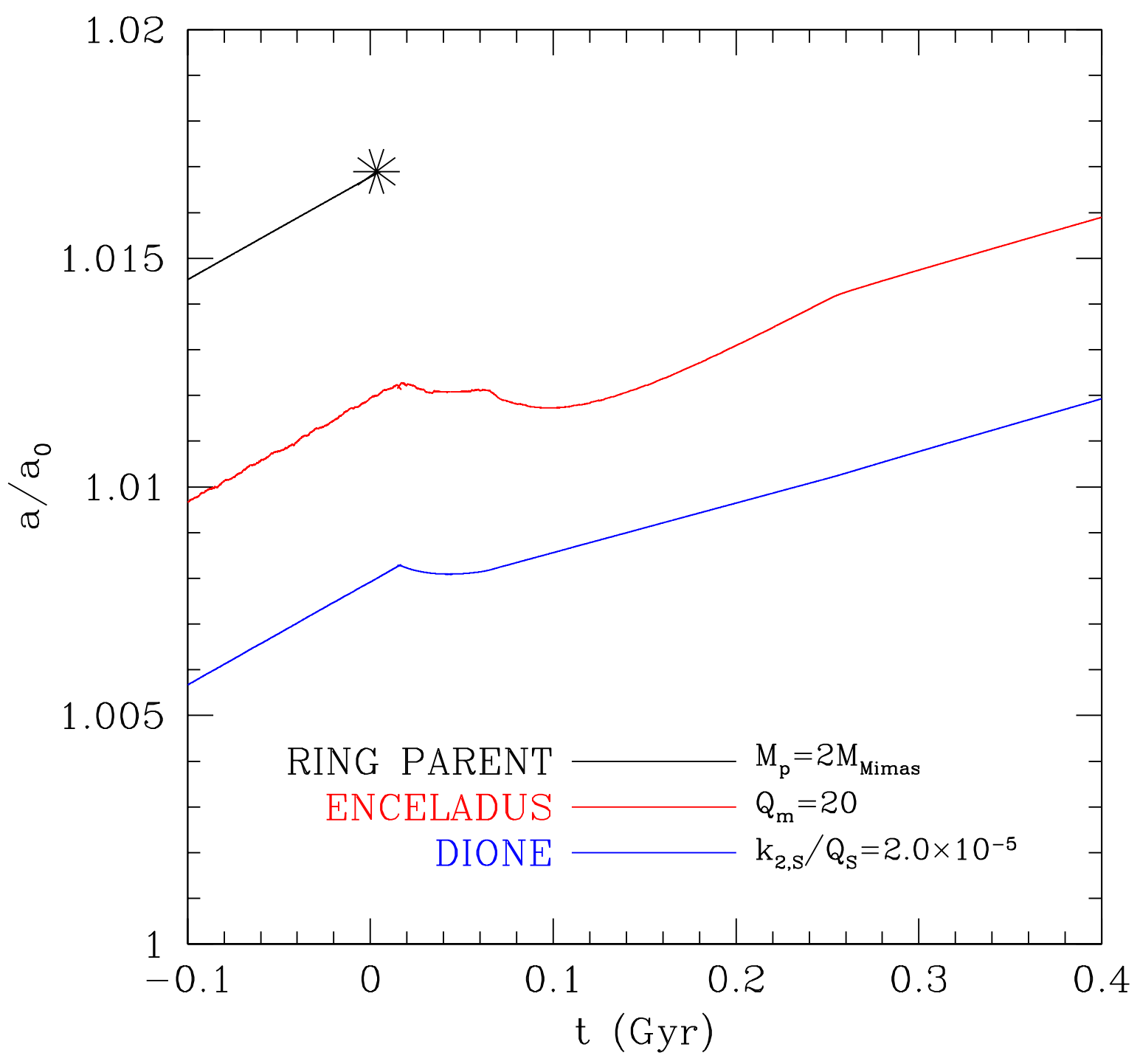

Figure 12: The evolution of the semi-major axes of Enceladus and Dione after the collisional disruption of the ring parent moon shown to occur at $t=0$. The tidal evolutionary growth of the semi-major axes stalls for 0.1 Gyr but Enceladus and Dione re-enter a MMR about 0.25 Gyr after the loss of the ring parent moon. 


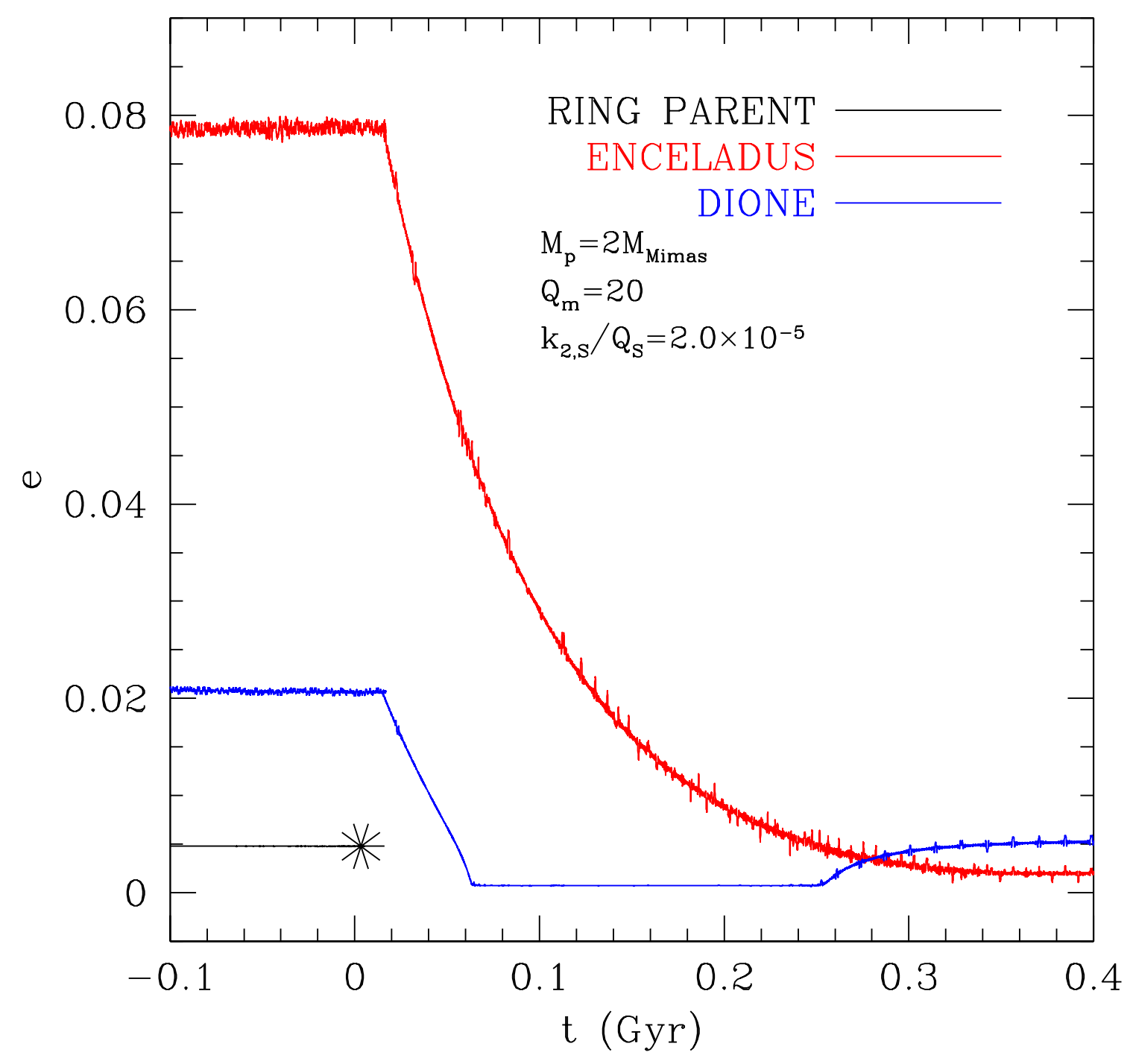

Figure 13: The evolution of the eccentricities of Enceladus and Dione after the collisional disruption of the ring parent moon at $t=0$. The eccentricities decay rapidly in the absence of the resonance but settle into values comparable to the currently measured values. While the choices of the satellite Love numbers and tidal dissipation functions $Q$ are only estimates, in this scenario Enceladus and Dione enter the current resonance and orbital configuration about $0.25 \mathrm{Gyr}$ after the collision. This is close to the timescale one expects for a newly formed Mimas to reach its current orbital radius after accretion of the ring debris onto the collision remnant. 
Carnahan, N.F., Starling, K.E., 1969. Equation of state for nonattracting rigid spheres. ”J. Chem. Phys.” 51, 635-636. URL: http://dx.doi. org/10.1063/1.1672048 doi 10.1063/1.1672048 arXiv:http://dx.doi.org/10.1063/1.1672048

Chambers, J.E., 1999. A hybrid symplectic integrator that permits close encounters between massive bodies. Mon. Not. R. Astr. Soc. 304 , 793-799. doi $10.1046 / j .1365-8711.1999 .02379 . x$

Charnoz, S., Canup, R.M., Crida, A., Dones, L., 2018. The Origin of Planetary Ring Systems. pp. 517-538. doi 10.1017/9781316286791.018

Charnoz, S., Dones, L., Esposito, L.W., Estrada, P.R., Hedman, M.M., 2009a. Origin and Evolution of Saturn's Ring System. Springer. chapter 17. p. 537. doi 10.1007/978-1-4020-9217-6_17

Charnoz, S., Morbidelli, A., Dones, L., Salmon, J., 2009b. Did Saturn's rings form during the Late Heavy Bombardment? Icarus $199,413-428$. doi $10.1016 /$ j.icarus.2008.10.019 arXiv:0809.5073

Charnoz, S., Salmon, J., Crida, A., 2010. The recent formation of Saturn's moonlets from viscous spreading of the main rings. Nature 465, 752-754. doi 10.1038/nature09096

Clisby, N., McCoy, B.M., 2006. Ninth and tenth order virial coefficients for hard spheres in d dimensions. J. Stat. Phys. 122, 15-57. URL: http://dx.doi.org/10.1007/s10955-005-8080-0 doi 10.1007/s10955-005-8080-0

Colwell, J.E., 1994. The disruption of planetary satellites and the creation of planetary rings. Planetary Space Sci. 42, 1139-1149. doi 10.1016/ 0032-0633(94)90013-2

Crida, A., Charnoz, S., 2012. Formation of Regular Satellites from Ancient Massive Rings in the Solar System. Science 338, 1196. doi 10.1126/ science.1226477, arXiv: 1301.3808

Ćuk, M., Dones, L., Nesvorný, D., 2016. Dynamical Evidence for a Late Formation of Saturn's Moons. Astrophys. J. 820, 97. doi 10.3847/ 0004-637X/820/2/97, arXiv: 1603.07071

Cuzzi, J.N., Burns, J.A., Charnoz, S., Clark, R.N., Colwell, J.E., Dones, L., Esposito, L.W., Filacchione, G., French, R.G., Hedman, M.M., Kempf, S., Marouf, E.A., Murray, C.D., Nicholson, P.D., Porco, C.C., Schmidt, J., Showalter, M.R., Spilker, L.J., Spitale, J.N., Srama, R., Sremčević, M., Tiscareno, M.S., Weiss, J., 2010. An evolving view of Saturn's dynamic rings. Science 327, 1470-. doi 10.1126/science.1179118

Cuzzi, J.N., Estrada, P.R., 1998. Compositional evolution of Saturn's rings due to meteoroid bombardment. Icarus 132, 1-35. doi 10.1006/icar . 1997.5863

Daisaka, H., Tanaka, H., Ida, S., 2001. Viscosity in a dense planetary ring with self-Gravitating particles. Icarus 154, 296-312. doi 10.1006/ icar.2001.6716

Dohnanyi, J.S., 1969. Collisional model of asteroids and their debris. J. Geo. Res. 74, 2531-2554. doi 10.1029/JB074i010p02531

Dones, L., 1991. A recent cometary origin for Saturn's rings? Icarus 92, 194-203. doi 10.1016/0019-1035(91) 90045-U

Dones, L., Chapman, C.R., McKinnon, W.B., Melosh, H.J., Kirchoff, M.R., Neukum, G., Zahnle, K.J., 2009. Icy Satellites of Saturn: Impact Cratering and Age Determination. p. 613. doi 10.1007/978-1-4020-9217-6_19

Dubinski, J., 1996. A parallel tree code. New Astron. 1, 133-147. doi 10.1016/S1384-1076(96) 00009-7 arXiv:astro-ph/9603097

Durisen, R.H., Bode, P.W., Cuzzi, J.N., Cederbloom, S.E., Murphy, B.W., 1992. Ballistic transport in planetary ring systems due to particle erosion mechanisms. II - Theoretical models for Saturn's A- and B-ring inner edges. Icarus 100, 364-393. doi 10.1016/0019-1035(92) 90106-H

Esposito, L., O'Callaghan, M., West, R., 1983. The structure of saturn's rings: Implications from the voyager stellar occultation. Icarus 56, 439 - 452. URL:http://www.sciencedirect.com/science/article/pii/0019103583901653 doi http://dx.doi.org/10.1016/ 0019-1035(83)90165-3

Esposito, L.W., 1986. Structure and evolution of Saturn's rings. Icarus 67, 345-357. doi 10.1016/0019-1035(86)90119-3

Estrada, P.R., Durisen, R.H., Cuzzi, J.N., Morgan, D.A., 2015. Combined structural and compositional evolution of planetary rings due to micrometeoroid impacts and ballistic transport. Icarus 252, 415-439. doi 10.1016/j . icarus.2015.02.005

Fuller, J., Luan, J., Quataert, E., 2016. Resonance locking as the source of rapid tidal migration in the Jupiter and Saturn moon systems. Mon. Not. R. Astr. Soc. 458, 3867-3879. doi 10.1093/mnras/stw609 arXiv: 1601.05804

Goldreich, P., 1965. An explanation of the frequent occurrence of commensurable mean motions in the solar system. Mon. Not. R. Astr. Soc. 130, 159. doi $10.1093 / \mathrm{mnras} / 130.3 .159$

Goldreich, P., Soter, S., 1966. Q in the solar system. Icarus 5, 375-389. doi 10.1016/0019-1035(66)90051-0

Goldreich, P., Tremaine, S., 1982. The dynamics of planetary rings. Ann. Rev. Astron. Astrophys. 20, 249-283. doi 10.1146/annurev . aa. 20. 090182.001341

Goldreich, P., Tremaine, S.D., 1978. The velocity dispersion in Saturn's rings. Icarus 34, 227-239. doi 10.1016/0019-1035(78) 90164-1

Harris, A.W., 1984. The origin and evolution of planetary rings, in: Greenberg, R., Brahic, A. (Eds.), IAU Colloq. 75: Planetary Rings, pp. $641-659$.

Hartmann, W.K., 1978. Planet formation - Mechanism of early growth. Icarus 33, 50-61. doi 10.1016/0019-1035(78)90023-4

Hedman, M.M., Nicholson, P.D., 2016. The B-ring's surface mass density from hidden density waves: less than meets the eye? Icarus 279 , 109-124. doi 10.1016/j.icarus.2016.01.007 arXiv: 1601.07955

Higa, M., Arakawa, M., Maeno, N., 1996. Measurements of restitution coefficients of ice at low temperatures. Planetary Space Sci. 44, 917-925. doi 10.1016/0032-0633(95)00133-6

Holsapple, K.A., 1993. The scaling of impact processes in planetary sciences. Annual Review of Earth and Planetary Sciences 21 , $333-373$. doi 10.1146/annurev.ea.21.050193.002001

Hyodo, R., Charnoz, S., 2017. Dynamical Evolution of the Debris Disk after a Satellite Catastrophic Disruption around Saturn. Astron. J. 154, 34. doi 10.3847/1538-3881/aa74c9 arXiv: 1705.07554

Hyodo, R., Charnoz, S., Ohtsuki, K., Genda, H., 2017. Ring formation around giant planets by tidal disruption of a single passing large Kuiper belt object. Icarus 282, 195-213. doi 10.1016/j.icarus.2016.09.012 arXiv:1609.02396

Jacobson, R.A., Antreasian, P.G., Bordi, J.J., Criddle, K.E., Ionasescu, R., Jones, J.B., Mackenzie, R.A., Meek, M.C., Parcher, D., Pelletier, F.J., Owen, Jr., W.M., Roth, D.C., Roundhill, I.M., Stauch, J.R., 2006. The Gravity Field of the Saturnian System from Satellite Observations and Spacecraft Tracking Data. Astron. J. 132, 2520-2526. doi $10.1086 / 508812$

Kraus, R.G., Senft, L.E., Stewart, S.T., 2011. Impacts onto $\mathrm{H}_{2} \mathrm{O}$ ice: Scaling laws for melting, vaporization, excavation, and final crater size. Icarus $214,724-738$. doi $10.1016 / \mathrm{j}$. icarus . 2011.05.016 
Lainey, V., Jacobson, R.A., Tajeddine, R., Cooper, N.J., Murray, C., Robert, V., Tobie, G., Guillot, T., Mathis, S., Remus, F., Desmars, J., Arlot, J.E., De Cuyper, J.P., Dehant, V., Pascu, D., Thuillot, W., Le Poncin-Lafitte, C., Zahn, J.P., 2017. New constraints on Saturn's interior from Cassini astrometric data. Icarus 281, 286-296. doi 10.1016/j.icarus.2016.07.014 $\operatorname{arXiv:1510.05870}$

Lainey, V., Karatekin, Ö., Desmars, J., Charnoz, S., Arlot, J.E., Emelyanov, N., Le Poncin-Lafitte, C., Mathis, S., Remus, F., Tobie, G., Zahn, J.P., 2012. Strong Tidal Dissipation in Saturn and Constraints on Enceladus' Thermal State from Astrometry. Astrophys. J. 752, 14. doi 10.1088/ 0004-637X/752/1/14 arXiv: 1204.0895

Lee, M.H., Peale, S.J., 2002. Dynamics and Origin of the 2:1 Orbital Resonances of the GJ 876 Planets. Astrophys. J. 567, 596-609. doi 10. $1086 / 338504$

Leinhardt, Z.M., Richardson, D.C., Quinn, T., 2000. Direct N-body Simulations of Rubble Pile Collisions. Icarus 146, 133-151. doi 10.1006/ icar.2000.6370 arXiv: astro-ph/9908221

Levison, H.F., Duncan, M.J., Zahnle, K., Holman, M., Dones, L., 2000. NOTE: Planetary Impact Rates from Ecliptic Comets. Icarus 143, 415-420. doi 10.1006/icar.1999.6313

Lissauer, J.J., Peale, S.J., Cuzzi, J.N., 1984. Ring torque on Janus and the melting of Enceladus. Icarus 58, 159-168. doi 10.1016/ 0019-1035(84) 90034-4

Lissauer, J.J., Squyres, S.W., Hartmann, W.K., 1988. Bombardment history of the Saturn system. J. Geo. Res. 93, 13776-13804. doi 10.1029/ JB093iB11p13776

Matson, D.L., Castillo-Rogez, J.C., Schubert, G., Sotin, C., McKinnon, W.B., 2009. The Thermal Evolution and Internal Structure of Saturn's Mid-Sized Icy Satellites. Springer. p. 577. doi 10.1007/978-1-4020-9217-6_18

Meyer, J., Wisdom, J., 2007. Tidal heating in Enceladus. Icarus 188, 535-539. doi $10.1016 / \mathrm{j}$. icarus.2007.03.001

Meyer, J., Wisdom, J., 2008. Tidal evolution of Mimas, Enceladus, and Dione. Icarus 193, 213-223. doi 10.1016/j .icarus .2007.09.008

Movshovitz, N., Nimmo, F., Korycansky, D.G., Asphaug, E., Owen, J.M., 2015. Disruption and reaccretion of midsized moons during an outer solar system Late Heavy Bombardment. Geophys. Res. Lett. 42, 256-263. doi 10.1002/2014GL062133

Murray, C.D., Dermott, S.F., 2000. Solar System Dynamics. Cambridge University Press.

Neveu, M., Rhoden, A.R., 2017. The origin and evolution of a differentiated Mimas. Icarus 296, 183-196. doi 10.1016/j.icarus.2017.06. 011

Ojakangas, G.W., Stevenson, D.J., 1986. Episodic volcanism of tidally heated satellites with application to Io. Icarus 66, 341-358. doi 10.1016/ 0019-1035(86)90163-6

Peale, S.J., 1999. Origin and evolution of the natural satellites. Ann. Rev. Astron. Astrophys. 37, 533-602. doi 10.1146/annurev . astro.37. 1.533

Rein, H., Lesur, G., Leinhardt, Z.M., 2010. The validity of the super-particle approximation during planetesimal formation. Astron. Astrophys. 511, A69. doi 10.1051/0004-6361/200912870 arXiv: 1001.0109

Richardson, D.C., Quinn, T., Stadel, J., Lake, G., 2000. Direct large-scale N-Body simulations of planetesimal dynamics. Icarus 143 , 45-59. doi 10.1006/icar.1999.6243

Robbins, S.J., Stewart, G.R., Lewis, M.C., Colwell, J.E., Sremčević, M., 2010. Estimating the masses of Saturn's A and B rings from high-optical depth N-body simulations and stellar occultations. Icarus 206, 431-445. doi $10.1016 / \mathrm{j}$. icarus.2009.09.012

Roche, E.A., 1847. Mémoire sur la figure d'une masse fluide, soumise à l'attraction d'un point éloigné. Acad. Sci. .Lett. Montpelier Mem. Section Sci. 1, 243-262.

Salmon, J., Charnoz, S., Crida, A., Brahic, A., 2010. Long-term and large-scale viscous evolution of dense planetary rings. Icarus 209, 771-785. doi $10.1016 /$ j.icarus.2010.05.030 arXiv:1006.0633

Sinclair, A.T., 1972. On the origin of the commensurabilities amongst the satellites of Saturn. Mon. Not. R. Astr. Soc. 160, 169. doi 10.1093/ mnras/160.2.169

Smith, B.A., Soderblom, L., Batson, R.M., Bridges, P.M., Inge, J.L., Masursky, H., Shoemaker, E., Beebe, R.F., Boyce, J., Briggs, G., Bunker, A., Collins, S.A., Hansen, C., Johnson, T.V., Mitchell, J.L., Terrile, R.J., Cook, A.F., Cuzzi, J.N., Pollack, J.B., Danielson, G.E., Ingersoll, A.P., Davies, M.E., Hunt, G.E., Morrison, D., Owen, T., Sagan, C., Veverka, J., Strom, R., Suomi, V.E., 1982. A new look at the Saturn system - The Voyager 2 images. Science 215, 504-537. doi 10.1126/science.215.4532.504

Speedy, R.J., 1998. Pressure and entropy of hard-sphere crystals. J. Phys.: Condens. Matter 10, 4387. URL: http://stacks.iop.org/ 0953-8984/10/i=20/a=006

Spencer, J.R., Pearl, J.C., Segura, M., Flasar, F.M., Mamoutkine, A., Romani, P., Buratti, B.J., Hendrix, A.R., Spilker, L.J., Lopes, R.M.C., 2006. Cassini Encounters Enceladus: Background and the Discovery of a South Polar Hot Spot. Science 311, 1401-1405. doi 10.1126/science. 1121661

Squyres, S.W., Reynolds, R.T., Cassen, P.M., 1983. The evolution of Enceladus. Icarus 53, 319-331. doi 10.1016/0019-1035(83) 90152-5

Tajeddine, R., Rambaux, N., Lainey, V., Charnoz, S., Richard, A., Rivoldini, A., Noyelles, B., 2014. Constraints on Mimas' interior from Cassini ISS libration measurements. Science 346, 322-324. doi 10.1126/science.1255299

Thomas, P.C., 2010. Sizes, shapes, and derived properties of the saturnian satellites after the Cassini nominal mission. Icarus 208 , 395-401. doi $10.1016 /$ j.icarus. 2010.01.025

Weaver, H.A., Lamy, P.L., 1997. Estimating the Size of Hale-Bopp's Nucleus. Earth Moon and Planets 79, 17-33. doi 10.1023/A: 1006220930046

Wisdom, J., 2008. Tidal dissipation at arbitrary eccentricity and obliquity. Icarus 193, 637-640. doi 10.1016/j.icarus.2007.09.002

Zahnle, K., Schenk, P., Levison, H., Dones, L., 2003. Cratering rates in the outer Solar System. Icarus 163, 263-289. doi 10.1016/ S0019-1035(03) 00048-4

Zhang, K., Nimmo, F., 2009. Recent orbital evolution and the internal structures of Enceladus and Dione. Icarus 204, 597-609. doi 10.1016/j. icarus.2009.07.007

Zhang, Z., Hayes, A.G., de Pater, I., Dunn, D.E., Janssen, M.A., Nicholson, P.D., Cuzzi, J.N., Butler, B.J., Sault, R.J., Chatterjee, S., 2019. VLA multi-wavelength microwave observations of Saturn's C and B rings. Icarus 317, 518-548. doi 10.1016/j.icarus.2018.08.014

Zhang, Z., Hayes, A.G., Janssen, M.A., Nicholson, P.D., Cuzzi, J.N., de Pater, I., Dunn, D.E., 2017a. Exposure age of Saturn's A and B rings, and 
the Cassini Division as suggested by their non-icy material content. Icarus 294, 14-42. doi 10.1016/j.icarus.2017.04.008

Zhang, Z., Hayes, A.G., Janssen, M.A., Nicholson, P.D., Cuzzi, J.N., de Pater, I., Dunn, D.E., Estrada, P.R., Hedman, M.M., 2017b. Cassini microwave observations provide clues to the origin of Saturn's C ring. Icarus 281, 297-321. doi 10.1016/j.icarus.2016.07.020

\section{Appendix A: Collisional N-body Algorithm}

Each particle in a system is represented as a hard massive sphere with a finite radius and frictionless surface with no internal angular momentum. The particles move under the influence of gravity and hard collisions with the equations of motion integrated using a second order leapfrog method. Gravitational forces for the system are computed using a parallelized treecode algorithm (Barnes and Hut, 1986, Dubinski, 1996). At each fixed step, the computed acceleration on each particle is used to change the particle velocity. At the step midpoint, a particle drifts at its current velocity and a nearest-neighbour finding algorithm is used to locate all particles within a fixed search radius that could possibly collide with that particle. All possible collisions are determined over the time interval and sorted in time. The particles are then moved forward in position to the time of the first collision in the sorted list. The velocities for the first pair of colliding particles (now touching) are adjusted by reversing the radial velocities thus conserving linear momentum through the collision. We assume the spheres have a frictionless surface so there is no transfer of angular momentum. After the first collision is complete, the neighborhood of this pair of particles is searched again for any new possible collisions. If new collisions are detected, they are added to the chronologically sorted list and the possible cascade of collisions caused by this first collision are detected and used to modify and re-sort the collision list. Once this is done, one then proceeds to the first collision in this new list and repeats the procedure again with the modified collision list. The sequence of collisions is therefore followed in order to the end of the time interval. Once the collision sequence is completed the particles are moved to their final position at the beginning of the next time interval, gravitational accelerations are computed again, velocities are updated and the entire procedure is repeated through the next timestep.

The algorithm is parallelized in the following way. The treecode method uses the concept of locally essential trees (Dubinski, 1996). In this method, the system is divided into rectangular subdomains in 3 dimensions each containing a unique subset of particles in the system mapped to a processor on a parallel supercomputer. A Barnes-Hut (1986) oct-tree structure is built for each subdomain used to compute local gravitational forces. To account for the external gravitational forces on particles from exterior subdomains, the relevant pieces of tree structures are imported to create a locally essential tree that contains enough information to compute the gravitational forces to the accuracy prescribed by the treecode algorithm.

The locally essential tree is also leveraged by the nearest-neighbour finding algorithm used for collision detection. Around a chosen particle, the tree structure is used to search the entire list of particles to find the nearest subset to test for possible collisions. It is hopelessly inefficient to search the entire list, so to speed up the process only the $\mathrm{N}$ nearest particles are examined where $N \approx 27$ typically. The oct-tree structure used to compute gravitational forces can be used efficiently to generate this list for each particle in a domain. The locally essential tree also contains particles from neighboring domains and so the procedure described above for determining and moving through a list of collisions can be applied in parallel in each subdomain with the small penalty of computing some collisions more than once for particles near the boundary. We find in practice this algorithm works well in parallel with as many as 1024 processors for the systems containing $10^{7-8}$ particles.

We incorporate collisional energy dissipation by incorporating inelasticity through a coefficient of restitution $\epsilon<$ 1. For inelastic collisions, the rebound velocity is reduced by a factor $\epsilon$ following other algorithms (e.g., Richardson et al. 2000). In practice, for the hypervelocity impacts simulated in this paper we assume the collisions are elastic with $\epsilon=1$ during the impact and disruption phase. When the debris from the collision disperses to form a ring, we use $\epsilon=0.9$ to dissipate energy.

With this algorithm, the possibility exists for missing some collisions of particles located near the domain boundaries. This pathology can lead to some errors in collision detection. A small fraction of particles can end up overlapping with their relative distance being less than a particle diameter. Any erroneous overlapping particles at the end of a time interval are assumed to be in a state of collision and their radial velocities are reversed. In practice, less than $0.1 \%$ of particles are overlapping between timesteps and if this problem arises the remedy is to reduce the time interval between steps until the fraction of overlappers is reduced to an acceptable level.

The collisional N-body code is available to other researchers by request. 


\section{Appendix B: Initial Conditions for Gravitating Rubble Piles}

In this appendix, we describe methods for setting up models of differentiated moons described as rubble piles composed of hard spheres of different densities to represent rock and ice. In condensed matter physics, systems of colliding hard spheres are used as idealized models for dense gases and liquids and can be described by a well-defined equation of state (EOS). The hard-sphere EOS depends on the packing fraction defined by $\eta=4 \pi / 3 r_{0}^{3} n$ with $n=N / V$ is the number density and $r_{0}$ is the radius of the hard spheres. The maximum value of $\eta$ is thought to be close to the maximum value of a system of hexagonally close-packed (HCP) spheres with a value $\eta_{\max }=\pi \sqrt{2} / 6 \approx 0.74$. The EOS is defined through the compressibility $Z \equiv P V / N k T$ : with $Z$ being some function of the packing fraction $\eta$. The compressibility can be expressed as a power series in $\eta$ :

$$
Z=1+\sum_{k=2}^{n_{\max }} b_{k} \eta^{k-1}
$$

where the $b_{k}$ are the virial coefficients, generally determined empirically through numerical simulations of hard sphere systems (e.g., Clisby and McCoy, 2006). Simpler analytic expressions, that are accurate to high order, exist such as the Carnahan-Starling (CS) EOS compressibility (Carnahan and Starling, 1969) is:

$$
Z=\frac{1+\eta+\eta^{2}-\eta^{3}}{(1-\eta)^{3}}
$$

We rewrite the equation of state in the form $P=\rho \sigma^{2} f(\rho)$ based on the definition of $\eta$ and $Z$ where $\rho$ is the density and $\sigma^{2}=k T / m$ is the velocity dispersion. There is a phase transition between $\eta=0.494$ and $\eta=0.545$ with approximately fixed $Z \approx 11.5$ where the hard-sphere systems freezes to a crystalline solid when using hexagonal close-packing. For $\eta<0.494$, we use $\mathrm{Z}$ as defined in equation B1. For $\eta>0.545$, the compressibility is accurately described by the empirical formula:

$$
Z=\frac{3}{1-z}-\frac{a(z-b)}{z-c}
$$

where $z=\eta / \eta_{\max }$ and $a=0.5935, b=0.708$ and $c=0.601$ as calculated by Speedy (1998).

To determine spherical equilibrium solutions for a self-gravitating system of hard spheres, we need only solve the equations of hydrostatic equilibrium to determine a density profile for a given initial central density for the rubble pile. Since there are no internal sources of energy, the solutions are isothermal. We therefore assume a MaxwellBoltzmann velocity dispersion $\sigma^{2}$ for the system of particles. The hydrostatic equations can be solved by choosing a central density with a guess for the velocity dispersion and integrating the equations to determine a density profile and total mass. The correct velocity dispersion can be determined by iteratively adjusting its value up or down depending on whether the total mass of a solution is less or greater than the desired value. We isolate the correct value of the velocity dispersion using a binary search. The end result is a spherical density profile and a single velocity dispersion. To set up the initial conditions for particle positions, we first lay out particles in hexagonally close-packed lattice of uniform density and carve out a sphere containing the desired number of particles. Given the mass profile $M(r)$ derived above we then adjust the particles radial positions according to $r \propto M^{1 / 3}$ so that radial mass profile is matched. Particle velocities are sampled from a Maxwell-Boltzmann distribution with the given velocity dispersion.

For a system with a single particle density, this procedure results in collisional hard sphere system in hydrostatic equilibrium. To model a differentiated moon, we apply a simple modification to the above procedure to set up a model with a rocky core and icy mantle. When initializing the unperturbed close-packed lattice of particles, we simply assign a higher mass to particles in the core according to the ratio of densities of rock to ice (approximately $3 \times$ ). The computation of the mass profile in hydrostatic equilibrium requires an adjustment to account for the greater particle density in the center but this is straightforward to implement within the iterative procedure. There is also a discontinuous change in the velocity dispersion of particles when moving from the rock core to the ice mantle to compute virial equilibrium. For systems with densities near maximum close-packing there can be discontinuities in the density profile within the core which are apparent in our models though the jump in density through the discontinuity is generally small.

We dynamically evolved the initial model to validate the equilibrium state. The density profile for the model moon used in this paper has been shown previously in Figure 1. The main points to note are the smooth drop off 
to zero density at the periphery compared with the ideal homogeneous model and the small discontinuity in density at $R=240 \mathrm{~km}$ due to the phase change. Figure B1 shows the relative change in potential, kinetic and binding energy for the model as a function of time for the $N=10 \mathrm{M}$ particle model. The model is slightly out of equilibrium with potential and kinetic energy being exchanged with amplitudes of $0.1 \%$ of the total binding energy. The system pulsates for several oscillations over the course of few hours but eventually damps down. The total binding energy in principle is a conserved quantity but integration errors cause it to drift by $0.01 \%$. In summary, using the hard-sphere EOS provides a useful away to set up rubble-pile models in hydrostatic equilibrium. We also plot the compressibility $Z=P / \rho \sigma^{2}$ as a function of the packing fraction measured from the $\mathrm{N}$-body model (Figure 6 ). The agreement between the measured and theoretical compressibility is very close showing the consistency of the equation of state with hydrostatic equilibrium.

The initial conditions code used to set up model moons in hydrostatic equilibrium is available to other researchers by request to the author. 


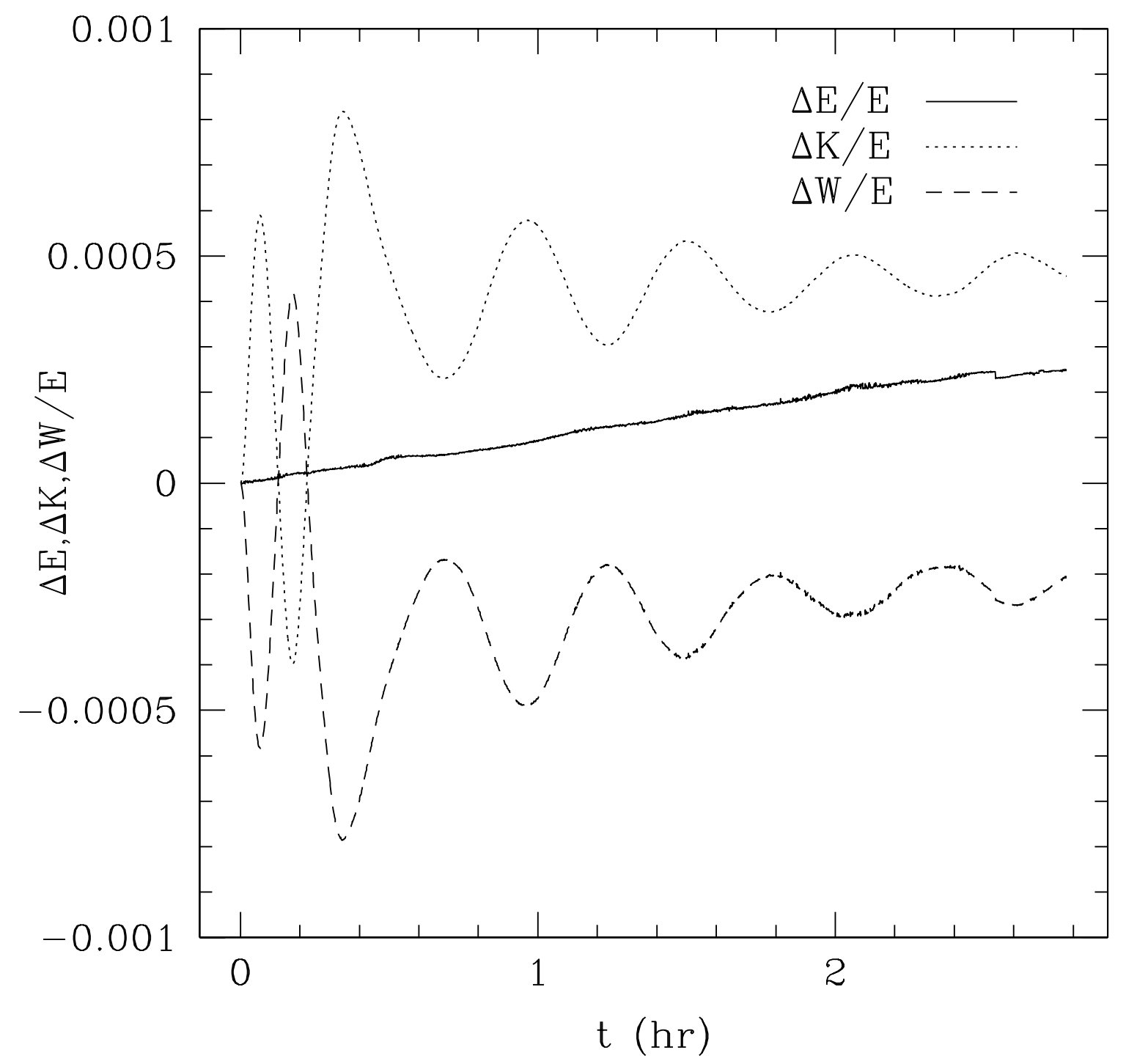

Figure B1: Energy evolution towards equilibrium for the initial spherical moon model. The system is evolved dynamically using the N-body code for several dynamical times. For a system in equilibrium, the total kinetic and potential energies should be constant in time. The system pulsates with energy being exchanged between kinetic and potential forms at the $0.1 \%$ level but the pulsations damp away after a few hours. The total energy of the system drifts by $0.02 \%$. The systems are almost in perfect virial equilibrium validating the input equation of state and the collisional N-body method. 


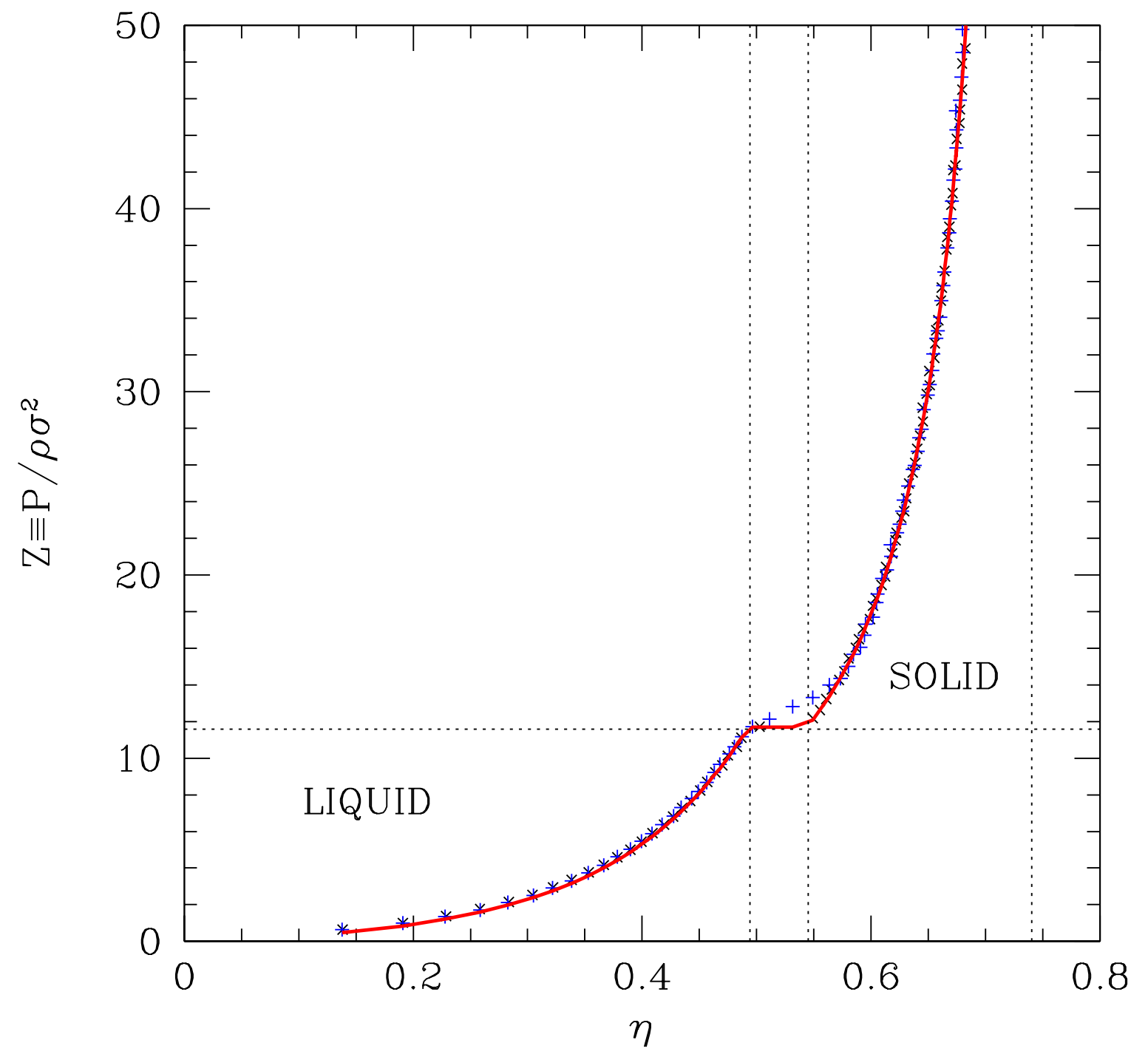

Figure B2: Compressibility measured from the equilibrium model at the initial (black crosses) and final times (blue crosses) compared with the theoretical expectation (red line). The agreement is very close and shows that both the equation of state and methods for computing the initial hydrostatic profile are consistent. 